\title{
Endothelial mitochondrial oxidative stress determines podocyte depletion in segmental glomerulosclerosis
}

\author{
Ilse Daehn, ${ }^{1}$ Gabriella Casalena, ${ }^{1}$ Taoran Zhang, ${ }^{1}$ Shaolin Shi, ${ }^{1}$ \\ Franz Fenninger, ${ }^{1}$ Nicholas Barasch, ${ }^{1}$ Liping Yu, ${ }^{1}$ Vivette D'Agati, ${ }^{2}$ \\ Detlef Schlondorff, ${ }^{1}$ Wilhelm Kriz, ${ }^{3}$ Borje Haraldsson, ${ }^{4}$ and Erwin P. Bottinger ${ }^{1}$
}

\begin{abstract}
1Division of Nephrology, Department of Medicine, and The Charles Bronfman Institute for Personalized Medicine, Icahn School of Medicine at Mount Sinai, New York, New York, USA. '2Department of Pathology, College of Physicians and Surgeons, Columbia University, New York, New York, USA.

3Department of Anatomy and Developmental Biology, Medical Faculty Mannheim, University of Heidelberg, Mannheim, Germany.

${ }^{4}$ Department of Molecular and Clinical Medicine - Nephrology, Institute of Medicine, University of Gothenburg, Gothenburg, Sweden.
\end{abstract}

Focal segmental glomerular sclerosis (FSGS) is a primary kidney disease that is commonly associated with proteinuria and progressive loss of glomerular function, leading to development of chronic kidney disease (CKD). FSGS is characterized by podocyte injury and depletion and collapse of glomerular capillary segments. Progression of FSGS is associated with TGF- $\beta$ activation in podocytes; however, it is not clear how TGF- $\beta$ signaling promotes disease. Here, we determined that podocyte-specific activation of TGF- $\beta$ signaling in transgenic mice and $\mathrm{BALB} / \mathrm{c}$ mice with Adriamycin-induced glomerulosclerosis is associated with endothelin-1 (EDN1) release by podocytes, which mediates mitochondrial oxidative stress and dysfunction in adjacent endothelial cells via paracrine EDN1 receptor type A (EDNRA) activation. Endothelial dysfunction promoted podocyte apoptosis, and inhibition of EDNRA or scavenging of mitochondrial-targeted ROS prevented podocyte loss, albuminuria, glomerulosclerosis, and renal failure. We confirmed reciprocal crosstalk between podocytes and endothelial cells in a coculture system. Biopsies from patients with FSGS exhibited increased mitochondrial DNA damage, consistent with EDNRA-mediated glomerular endothelial mitochondrial oxidative stress. Our studies indicate that segmental glomerulosclerosis develops as a result of podocyte-endothelial crosstalk mediated by EDN1/EDNRA-dependent mitochondrial dysfunction and suggest that targeting the reciprocal interaction between podocytes and endothelia may provide opportunities for therapeutic intervention in FSGS.

\section{Introduction}

Chronic kidney disease (CKD) affects more than $10 \%$ of the US population (1). CKD may lead to end-stage renal disease and is a major risk factor for cardiovascular disease and mortality $(2,3)$. Glomerular pathology is the hallmark in most CKD cases, including those associated with diabetes and hypertension $(1,4)$. Glomeruli are the functional filtration unit composed of a capillary network of endothelial cells and mesangial cells, separated from podocytes by a basement membrane $(5,6)$. Podocyte injury and loss contribute to proteinuria and glomerulosclerosis (7-12), while a role of endothelial injury remains relatively unexplored (13-15). Podocytes regulate endothelial cell growth and survival via VEGFA and angiopoietin-1 (ANG1), and loss of VEGFA or ANG1 is associated with increased endothelial damage and apoptosis and proteinuria $(16,17)$. Glomerular endothelial cells are highly specialized with fenestrae and a luminal glycocalyx layer $(5,14,18)$ that contributes to the filtration barrier $(13,19)$. In addition, certain forms of glomerular injury, including diabetes, cause endothelial dysfunction characterized by increased ROS, alterations in vasoreactivity, coagulation, and inflammation (5).

We have previously reported that TGF- $\beta$ induces podocyte apoptosis and depletion in transgenic mice and in cultured podocytes and leads to progressive glomerulosclerosis (20). TGF- $\beta$ and TGF- $\beta$ receptors are typically upregulated in podocytes in

Conflict of interest: The authors have declared that no conflict of interest exists. Citation for this article: J Clin Invest. 2014;124(4):1608-1621. doi:10.1172/JCI71195. experimental models of glomerulosclerosis and human glomerulosclerosis (21-26). Here, we used a constitutively active TGF- $\beta$ type I receptor (Tgfrb1) mutant for podocyte-specific, ligandindependent activation of TGF- $\beta$ signaling in a doxycyclineinducible (Dox-inducible) transgenic mouse model (27). Our results demonstrate a previously unrecognized dependence of podocyte dysfunction and depletion in glomerulosclerosis on podocyteendothelial crosstalk. Thus, podocyte-generated endothelin-1 (EDN1) causes endothelial mitochondrial oxidative damage via endothelin receptor type A (EDNRA), which was required for podocyte loss and glomerulosclerosis. This podocyte-endothelial crosstalk presents new therapeutic opportunities, including the use of mitochondrial-targeted ROS scavengers and endothelin antagonists to prevent endothelial dysfunction-dependent podocyte depletion and thereby glomerulosclerosis.

\section{Results}

Activation of podocyte-selective TGF $\beta R 1$ signaling induces progressive segmental glomerulosclerosis with albuminuria in transgenic mice. To examine the effects of TGF- $\beta$ signaling selectively in podocytes, we generated transgenic mice (referred to herein as PodTgfbr1 mice; described in the Methods), in which robust podocyte-specific expression of a ligand-independent, constitutively active TGFßR1 mutant $\left(\right.$ Tgfbr $\left.{ }^{A A D}\right)(28,29)$ can be induced within 24 hours by Dox chow feeding (Supplemental Figure 1, A and $\mathrm{B}$; supplemental material available online with this article; doi:10.1172/JCI71195DS1). Activation of TGFßR1-regulated 
SMAD2/SMAD3 signaling specifically in podocytes was confirmed by nuclear translocation of SMAD2/3 proteins (30) and exclusive colocalization with Wilms tumor antigen 1 (WT1), a podocyte-specific nuclear transcription factor (Figure 1, A and B). Figure $1 \mathrm{C}$ depicts the colocalization of SMAD2/3 and WT1 labeling in podocyte nuclei.

Dox-induced TGF $\beta$ R1 signaling in podocytes led to progressive increase of albuminuria by day 4 and of serum creatinine by day 7 (Figure 1D). Dox treatment also induced segmental glomerulosclerosis by day 7 (Figure $1 \mathrm{G}$ ) and global glomerulosclerosis with tubulointerstitial fibrosis by day 14 (Figure $1 \mathrm{H}$ ). Other glomerular cell lesions included transient mesangial cell proliferation between day 1 and day 4 (Figure 1F and Supplemental Figure 1C). TUNEL and WT1 double-positive apoptotic podocytes were detected by day 4 and loss of podocytes was detected by day 7 and day $14(\sim 25 \%$ and $\sim 40 \%$ reduction compared with baseline, respectively) (Figure 1I). Interestingly, endothelial cell protrusions and endothelial vesicle shedding were the first prominent ultrastructural defects by day 4 of Dox treatment (Figure 1J), followed by podocyte foot process effacement and membrane ruffling adjacent to abnormal endothelial cells by day 7 (Figure $1 \mathrm{~K}$ ).

In a survival experiment, 2 groups of 20 PodTgfbr 1 mice each were maintained on regular chow (control) or Dox chow continuously for up to 30 weeks. Five animals $(25 \%)$ in the Dox chow group required euthanasia due to moribund appearance beginning after 5 weeks on Dox, compared with none $(0 \%)$ among control mice (Supplemental Figure 2A). Of note, all moribund mice fed Dox chow manifested ascites and edema indicative of progressive kidney disease with high-grade albuminuria. To determine the phenotype parameters at which TGF $\beta$ R1-induced glomerulosclerosis became irreversible, Dox chow feeding was discontinued after day 7 , day 10 , or day 14 , and the PodTgfbr 1 mice then received regular chow for up to 21 additional days. Dox withdrawal at day 7 and day 10 was followed by normalization of elevated albumin/creatinine ratio (ACR) levels and normal glomerular histology after 10 days of Dox withdrawal (Figure 2, A and B). In contrast, Dox withdrawal after 14 days was associated with persistent highgrade albuminuria and advanced glomerulosclerosis, even after 21 days of Dox withdrawal (Figure 2, A and C), suggesting the threshold for irreversibility and PodTgfbr 1 transgene-independent progression of glomerulosclerosis and tubulointerstitial fibrosis occurred between $25 \%$ and $40 \%$ estimated podocyte depletion, similar to previous reports (31). Finally, to examine whether onset of TGF $\beta$ R 1 signaling in podocytes and progressive glomerulosclerosis in PodTgfbr1 mice resulted in increase of systemic blood pressure (BP), we monitored systolic and diastolic BPs at least twice daily in conscious animals on Dox chow by telemetry during a 14-day observation interval. Compared to baseline readings obtained over 2 days prior to initiation of Dox chow, systolic and diastolic BP as well as heart rate were not significantly changed during 14 days of Dox administration, which were characterized by development of proteinuria and progressive glomerulosclerosis (Supplemental Figure 2B).

Together, these results suggest that after activation of TGF $\beta$ R1/ SMAD signaling in podocytes, endothelial cell injury and mesangial cell proliferation preceded manifestations of podocyte injury, including foot process effacement and onset of progressive podocyte depletion and glomerulosclerosis associated with progressive renal insufficiency, albuminuria, and death. The progressive glomerulosclerosis phenotype associated with endothelial mitochon- drial dysfunction caused by podocyte-specific TGF $\beta$ R 1 signaling was not associated with systemic BP changes and progressed further autonomously and in a podocyte Tgfbr 1 transgene-independent manner when initial depletion of $25 \%$ to $40 \%$ of glomerular podocytes was surpassed.

TGF- $\beta$ signaling in podocytes decreases mitochondrial function and induces oxidative stress lesions in glomeruli prior to manifestation of podocyte injury. To begin to understand the molecular mechanism underlying the progressive glomerulosclerosis in PodTgfbr1 mice, we performed genome-wide glomerular gene expression and pathway analyses that indicated suppression of mitochondrial electron transport chain and antioxidant genes (Supplemental Table 1). QPCR analysis confirmed that expression of the mitochondrial antioxidant gene superoxide dismutase- 2 (Sod2) and selected mitochondrial electron transport chain genes was decreased within 1 day of Dox treatment (Figure 3A). Glomerular oxygen consumption rate (OCR), a measure of mitochondrial respiration reserve capacity, was significantly reduced by day 4 on Dox, with further subsequent decreases (Figure 3B). In contrast, reduction of OCR in the tubular fractions was modest and became significant only after day 14, when high-grade albuminuria was present (Figure 3B). 3-Nitrotyrosine, a marker of oxidative stress-induced protein damage in tissues $(32,33)$, was absent in controls (Figure 3C) but detectable in PodTgfbr1 glomeruli by day 4 of Dox treatment (Figure 3D). Accumulation of oxidative mitochondrial DNA ( $m t D N A)$ lesions has been associated with mitochondrial dysfunction (34). Quantitative analysis of oxidative DNA lesions by QPCR demonstrated a significant accumulation of mtDNA but not nuclear DNA lesions in glomeruli from Dox-treated PodTgfbr1 mice (Figure 3E). Consistent with these findings, 8-oxoguanine (8-oxoG; also known as 8-hydroxyguanine or OH8Gua), a common DNA modification resulting from ROS (35), accumulated specifically in mitochondria of glomeruli of PodTgfor 1 mice after 2 days on Dox (Figure 3F and Supplemental Figure 3) and in tubules after 7 days, concomitant with onset of high-grade albuminuria (Supplemental Figure 3). Moreover, 8-oxoG excretion in the urine was increased by day 4, likely originating from glomerular injury, and rose progressively thereafter, associated with glomerular and tubular injury (Figure 3G and Supplemental Figure 3). To confirm that the root cause of mitochondrial oxidative DNA damage was due to Dox-induced podocyte-specific TGF $\beta$ R1 signaling, mice were cotreated with LY364947, a selective TGF $\beta$ R1 kinase inhibitor. LY364947 prevented nuclear translocation of SMAD2/3 in podocytes when administered concomitantly with Dox chow (data not shown). When LY364947 was administered, starting only after day 4 of Dox treatment (when mitochondrial oxidative damage was typically established), glomerular 8-oxoG accumulation (Figure $3 \mathrm{H}$ ) and serum creatinine elevation $(0.21 \pm 0.01 \mathrm{mg} / \mathrm{dl}$ vs. $0.54 \pm 0.08 \mathrm{mg} / \mathrm{dl})$, which typically manifests by day 7 , were significantly reduced compared with that after treatment with Dox alone for 7 days.

Together, these results demonstrate that podocyte-selective activation of TGF- $\beta$ signaling is the root cause of rapid mitochondrial dysfunction and oxidative mtDNA damage in glomeruli of PodTgfbr 1 mice. Importantly, glomerular oxidative mtDNA damage and renal lesions were reversed when TGF $\beta$ R1 activation was inhibited after oxidative mtDNA damage was already established (day 4) but prior to onset of podocyte depletion and foot process effacement (day 7 ). 
A

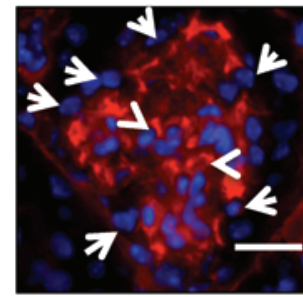

B
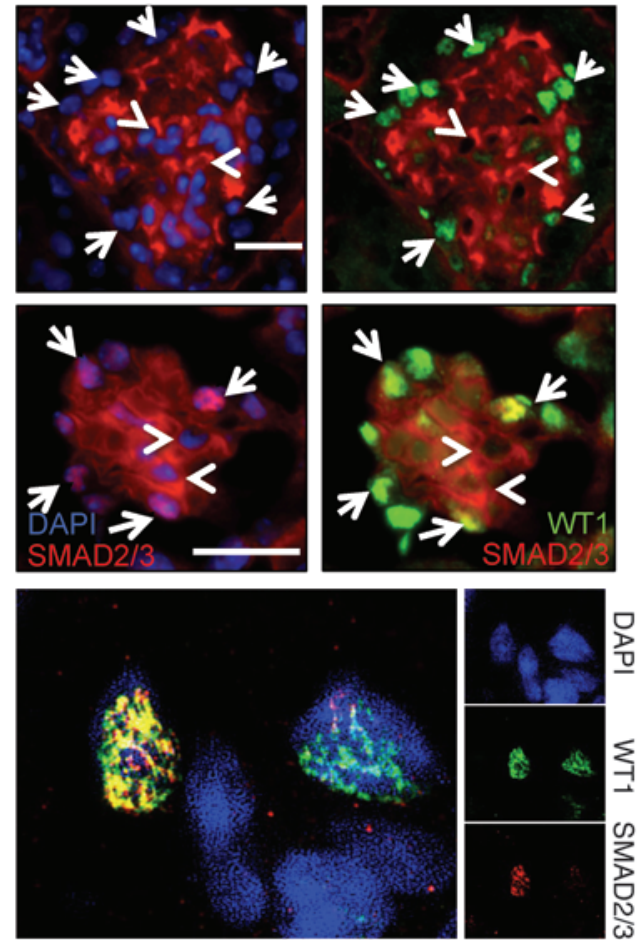

D

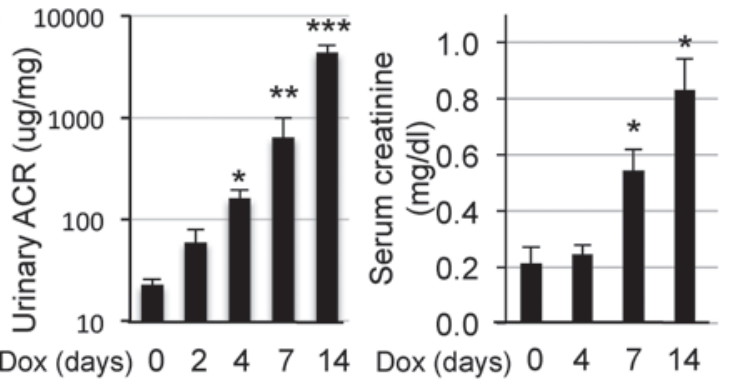

E
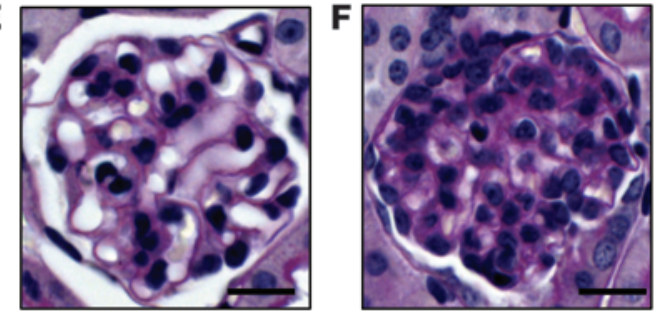

G

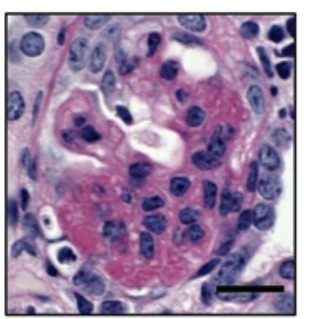

I
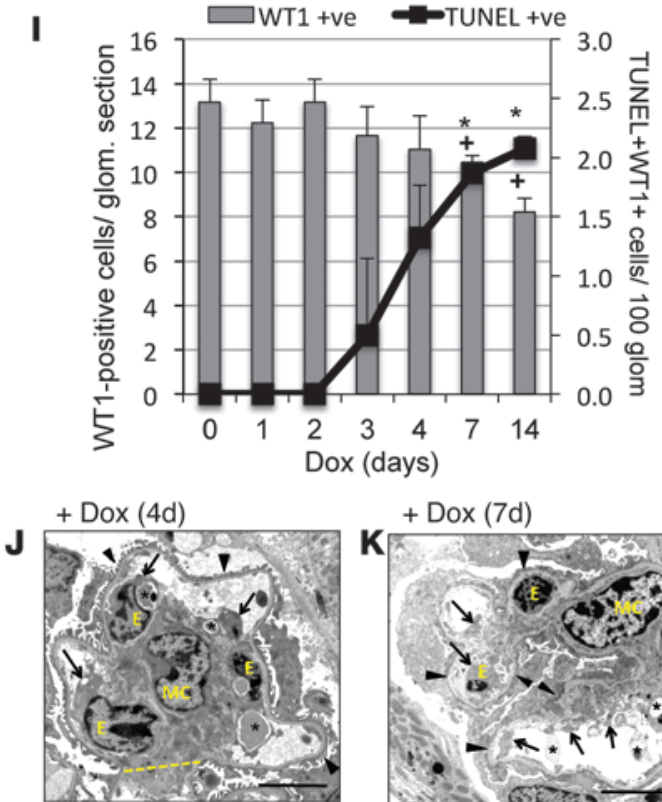
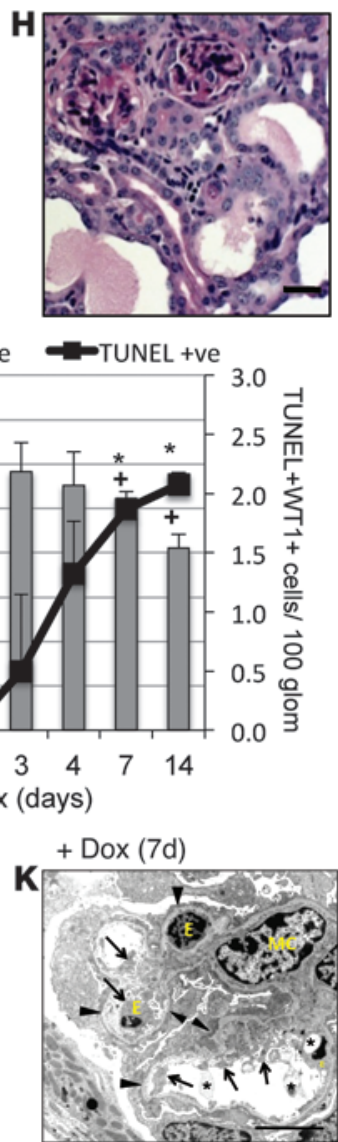

Figure 1

Activation of podocyte-specific TGF $\beta$ R1 induces podocytopathy with progressive glomerular disease and renal failure. Representative glomerular triple-immunofluorescence staining in PodTgfbr1 mice on (A) regular chow and (B) after 2 days of Dox chow, showing SMAD2/3 and DAPI and SMAD2/3 and WT1 localization. Arrows depict nuclear WT1 and DAPI in podocytes in A and colocalization with SMAD2/3 in B. Arrowheads denote cytoplasmic SMAD2/3 staining. (C) Superresolution image of SMAD2/3 (red) specifically localized to WT1- (green) and DAPI-positive (blue) cells. (D) ACR in PodTgfbr1 mice treated with Dox (days $0-14 ; n=6$ mice per group) and serum creatinine in Dox-treated PodTgfbr1 mice $(n=5$ mice per group; mean \pm SEM). (E-H) Histopathology stain (PAS) of PodTgfbr1 mice: (E) control mice without Dox, (F) day 4 of Dox, (G) day 14 of Dox, and $(\mathbf{H})$ day 14 of Dox. (I) Podocyte number (gray bars) and podocyte apoptosis (black line) of Dox-treated PodTgfbr1 mice (mean \pm SD; $>50$ glomerular profiles per mouse; $>5$ mice per time point). (J) Ultrastructural analysis by electron microscopy of day 4 Dox PodTgfbr1 mice. Glomerular area with mesangial expansion and endothelial cells (E) that protrude (arrows) and shed material (asterisks) into capillary lumens. Podocytes show normal foot process pattern (arrowheads). MC, mesangial cell. (K) Electron microscopy images of day 7 Dox PodTgfbr1 mice. Glomerular area with similar mesangial and endothelial changes. Podocytes show extensive foot process effacement (arrowheads). Scale bar: $50 \mu \mathrm{m}$ (A, B, and E-H); $5 \mu \mathrm{m}(\mathbf{J}$ and $\mathbf{K})$. Original magnification, $\times 63(\mathbf{A}, \mathbf{B}$, and $\mathbf{E}-\mathbf{G}) ; \times 100(\mathbf{C}) ; \times 20(\mathbf{H}) .{ }^{*} P<0.05,{ }^{* \star} P<0.01,{ }^{\star * \star} P<0.001$ versus controls.

Mitochondrial oxidative stress and dysfunction localize to glomerular endothelial cells in murine models of podocyte injury and in buman focal segmental glomerulosclerosis. Surprisingly, the oxidative mtDNA damage marker 8-oxoG was not colocalized with the podocyte marker synaptopodin (Figure 4B) but was detectable only in CD31positive endothelial cells in glomeruli of PodTgfbr1 mice (Figure 4A). The seemingly paradoxical coincidence of podocyte-selective acti- vation of TGF $\beta$ R1 signaling documented by SMAD2/3 translocation in podocytes and the accumulation of 8 -oxoG in glomeruli endothelia was confirmed in additional models of podocyte-initiated glomerulosclerosis, including podocyte-specific knockout of the microRNA-processing enzyme Dicer (ref. 36 and Supplemental Figure 4A), and BALB/c mice subjected to an Adriamycin (AD) podocyte injury model (ref. 37 and Supplemental Figure 4, B-D). 

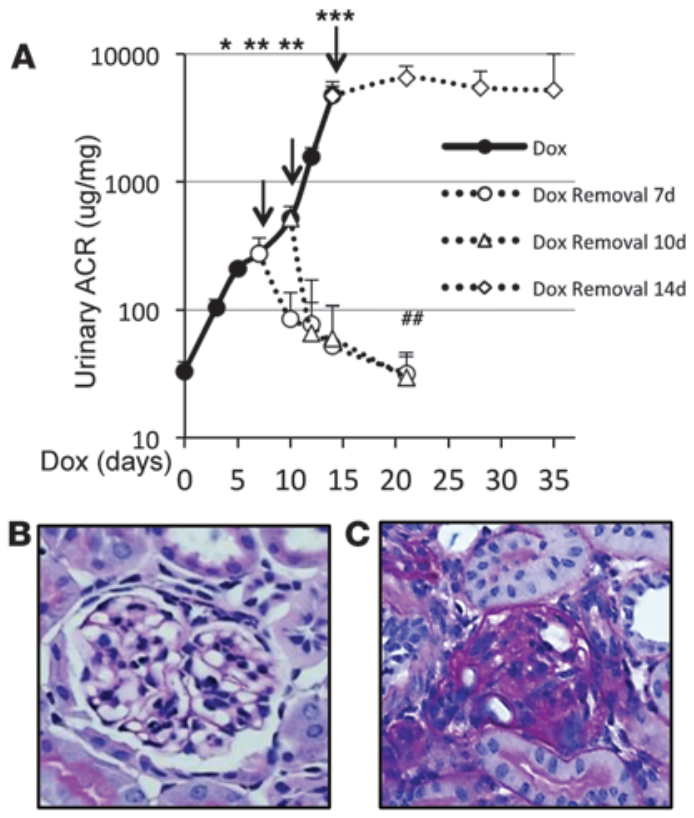

\section{Figure 2}

TGF $\beta$ R1 signaling in podocyte-induced albuminuria is reversible at days 7 and 10 but not day 14 of Dox treatment. (A) ACR in PodTgfbr1 mice treated with Dox (days 0-14) (>10 mice per time point). Arrows indicate Dox withdrawal at day 7 , day 10 , or day 14 for mice returned to normal diet ( $n=6$ per time point; mean \pm SEM). (B) Representative image of a PodTgfbr1 mouse with 2 weeks of recovery after days 10 of Dox withdrawal. (C) Representative image of a PodTgfbr1 mouse with 3 weeks of recovery after days 14 of Dox withdrawal. ${ }^{*} P<0.05$, ${ }^{\star *} P<0.01$, ${ }^{* * *} P<0.001$ versus controls; \#\# $P<0.01$ versus time of Dox withdrawal, i.e., 7 days and 10 days. Original magnification, $\times 40$ (B and C).

Similar findings of accumulation of 8-oxoG in endothelial cells of segmental sclerosis areas were observed in a series of human kidney biopsies from patients with focal segmental glomerular sclerosis (FSGS) (Figure 4C; clinical data available in Supplemental Table 2) but not in normal control human kidney biopsies (data not shown).

Thus, TGF- $\beta$ signaling in podocytes causes subsequent mitochondrial oxidative stress, specifically in glomerular endothelial cells (not in podocytes), consistent with the morphologic evidence for endothelial cell injury manifest within 4 days of Dox treatment, as shown by electron microscopy, prior to manifestations of characteristic podocyte defects by day 7 of Dox treatment, including foot process effacement and podocyte depletion (see Figure 1, J and K). Endothelial cell stress and injury were further validated with a dramatic 6-fold (day 4) and 8-fold (day 7) increase of endothelial cell apoptosis, as assessed by quantitation of glomerular cells identified as isolectin- and TUNEL double-positive glomerular cells in Dox-fed PodTgfbr 1 mice compared with control PodTgfbr1 mice (Figure 4, D and E).

TGF $\beta R 1$ signaling induces de novo synthesis of EDN1 precursor preproendothelin-1 in podocytes and expression of EDNRA in endothelial cells in PodTgfbr1 mice and a novel podocyte-endothelial coculture system. Comparison of glomerular microarray profiles determined at day 1, day 4, day 7, and day 14 in PodTgfbr 1 mice on Dox or control chow indicated that cognate ligand-receptor pair $E d n 1$ (also known as ET-1) and Ednra (also known as ETAR or ETA) mRNA were increased during the first week of Dox treatment in PodTgfbr1 mice compared with baseline (data not shown). We confirmed an increase of Ednra mRNA (Figure 5A). Similarly, Edn1 mRNA was increased in glomeruli after Dox by 10\% (1 day), 22\% (day 4), and $43 \%$ (day 7) compared with that in controls. To define precisely the glomerular cell type(s) responsible for increased Ednra and Edn1 mRNA synthesis, we immunostained glomerular sections of PodTgfbr1 mice with EDN1 precursor protein preproendothelin-1 antibody or EDNRA antibody, respectively. Intracellular preproendothelin-1 was absent in control PodTgfbr 1 mice but became readily detectable in a podocyte-like distribution after only 1 day of Dox treatment (Figure 5B). When PodTgfbr1 mice were cotreated with TGF $\beta$ R1 kinase inhibitor LY364947 and Dox for 4 days, preproendothelin-1 synthesis was prevented, compared with that when mice were treated with Dox alone (Figure 5C), confirming that rapid preproendothelin-1 synthesis was indeed induced by activation of TGF $\beta R 1$ signaling in podocytes.

In contrast, while EDNRA transmembrane receptor was not present in glomeruli of control PodTgfbr1 mice, EDNRA became detectable by colocalization with CD31-positive glomerular endothelial cells (Figure 5D) but remained absent in synaptopodinexpressing podocytes (Figure 5E). Increased endothelial EDNRA expression was confirmed in another podocyte injury model, $\mathrm{AD}$-treated BALB/c mice (Supplemental Figure 4D, bottom panel). Consistent with our observations in these experimental animal models, EDNRA colocalized with 8-oxoG in segmental sclerosis lesions in human kidney biopsies of patients with FSGS (Figure 5F), and 8-oxoG was also colocalized with endothelial CD31 (see Figure 4C). EDNRA and 8-oxoG were not detectable in glomeruli of normal human kidney (data not shown).

To examine further the regulation and functional role(s) of the EDN1/EDNRA-mediated podocyte-to-endothelial cell crosstalk suggested by findings in two podocyte injury models and human FSGS, we generated and characterized conditionally immortalized Dox -inducible double-transgenic Nphs2-2-rtTA.tet-O-Tgfbr $1^{A A D}$ podocyte (POD) cell lines from PodTgfbr1 mice (see Methods). Similar to TGF$\beta 1$ ligand, Dox treatment of POD cells activated SMAD phosphorylation and nuclear translocation (Supplemental Figure 5, A and B; see complete unedited blots in the supplemental material) and had no significant effect on mitochondrial oxidative phosphorylation or glycolysis in POD cells (Supplemental Figure 5, C and D). However, activation of TGF- $\beta$ signaling by Dox treatment in POD cells was associated with increased $E d n 1$ mRNA (Figure 5G) and release of mature EDN1 peptide in POD culture supernatants (PSNs) (Figure 5H). In contrast, Ednra mRNA levels were barely detectable and unchanged in untreated or Dox- or TGF- $\beta 1$-treated POD cells (data not shown). Next, we began to explore the signaling mechanisms that underlie stimulation of EDN1 synthesis by TGF $\beta$ R1 signaling in podocytes. Edn1 mRNA and EDN1 peptide release, characteristic of Dox-treated POD cells or TGF- $\beta$-treated WT podocytes, was prevented by TGF $\beta$ R1 kinase inhibitor LY364947 (3 $\mu \mathrm{M})$ (Figure 5, F and G, and Supplemental Figure 5, E and F). Baseline levels of $E d n 1 \mathrm{mRNA}$ and peptide release were lower in Smad2 KO or Smad2/3 double KO (DKO) podocytes compared with those in WT podocytes (Supplemental Figure 5, E and F). TGF- $\beta$ treatment stimulated Edn1 mRNA levels and peptide release in WT podocytes but had no effect in Smad2 KO or Smad2/3 DKO podocytes (Supplemental Figure 5, E and F). These findings suggest that TGF- $\beta$ stimulates Edn1 mRNA synthesis and peptide release via its canonical SMAD2/3 signaling pathway(s) in podocytes in vitro. 

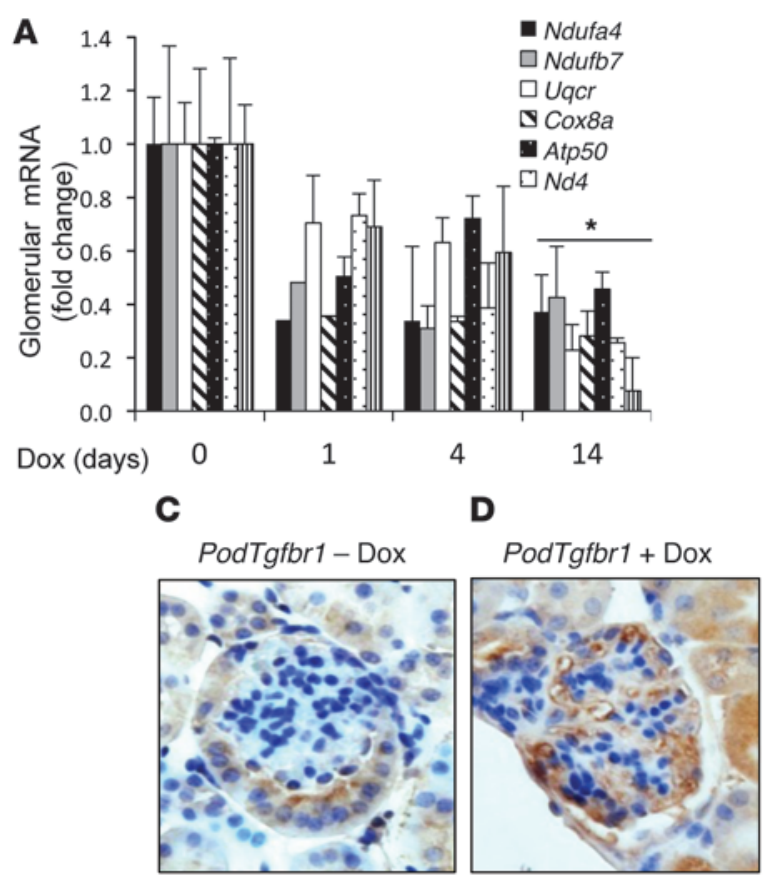

F $\quad$ 8-oxoG
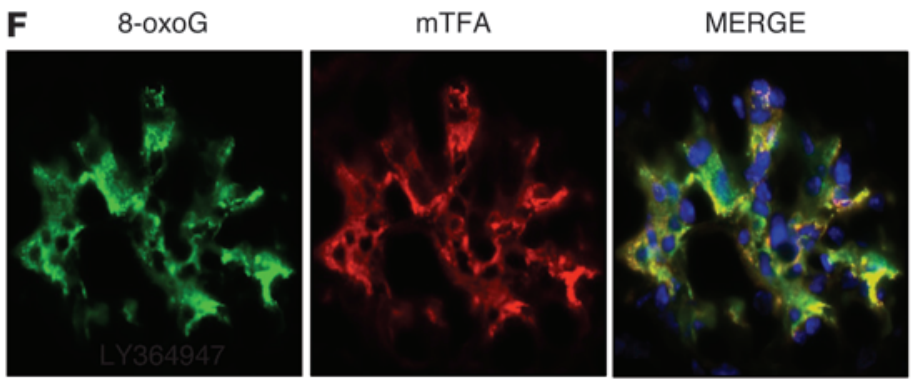

H

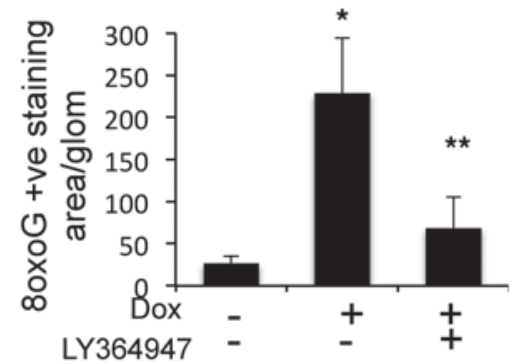

$\begin{array}{lllllll}\text { B } & \text { Dox (days) } & 0 & 14 & 4 & 7 & 14\end{array}$

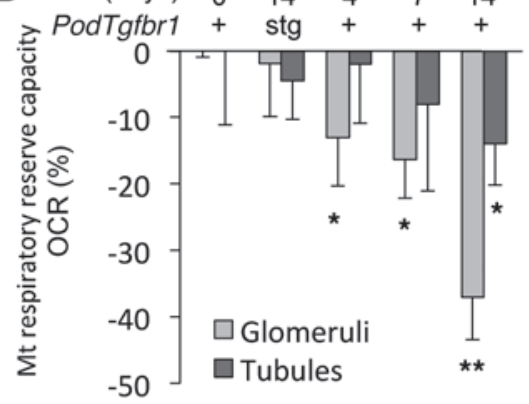

E

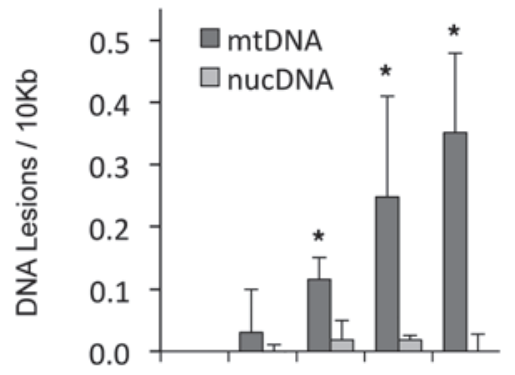

$\begin{array}{llllll}\text { Dox (days) } & 0 & 14 & 4 & 7 & 14\end{array}$

PodTgfbr1 + stg +++

G

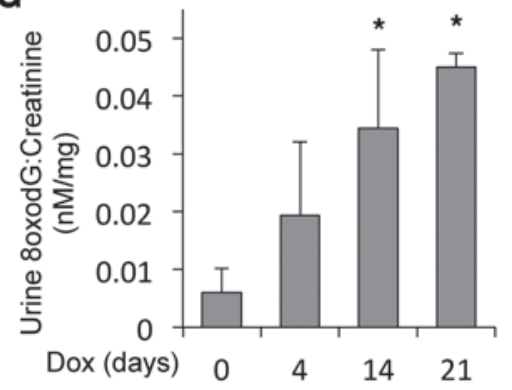

\section{Figure 3}

TGF- $\beta$ signaling in podocytes decreased mitochondrial genes and function and increased mtDNA damage in glomerular epithelial cells. (A) mRNA expression of mitochondrial proteins in isolated glomeruli from PodTgfbr1 mice after Dox for 0 to 14 days ( $n=3$; mean \pm SEM). (B) Mitochondrial respiratory reserve capacity was measured by OCR of isolated glomeruli and tubules in single transgenic mice (stg) or double-transgenic PodTgfbr1 mice (+) treated with Dox for the days indicated (values are mean percentage reduction in OCR \pm SEM; $n=8$ mice per time point). (C) Immunoperoxidase detecting 3-nitrotyrosine in control untreated PodTgfbr1 (-Dox) and (D) day 4 Dox-treated PodTgfbr1 (+Dox) mice. (E) Quantification of lesion frequencies in mtDNA and nuclear DNA by QPCR in isolated glomeruli of stg or PodTgfbr1 mice treated with Dox for up to day 14 relative to untreated day 0 controls $(n=6$; mean \pm SEM relative amplification normalized to nondamaged day 0 controls). (F) Representative images of double immunofluorescence detection of 8-oxoG (green) and mitochondrial transcription factor A (mTFA; red) in glomeruli of a day 4 Dox-treated PodTgfbr1 mouse. (G) Urine 8-oxodG relative to urine creatinine in PodTgfbr1 mice left untreated (control) or treated with Dox for up to 21 days $(n=6$; mean \pm SEM). (H) Quantification of 8-oxoG staining of glomeruli from Dox control PodTgfbr1 mice, mice fed Dox chow for 7 days, or Dox-fed PodTgfbr1 mice treated with $1 \mathrm{mg} / \mathrm{kg}$ LY364947 from day 4 to day 7 (mean \pm SEM). ${ }^{*} P<0.05,{ }^{* *} P<0.01$ versus controls. Original magnification, $\times 40(\mathbf{C}$ and $\mathbf{D}) ; \times 100(\mathbf{F})$. 
A

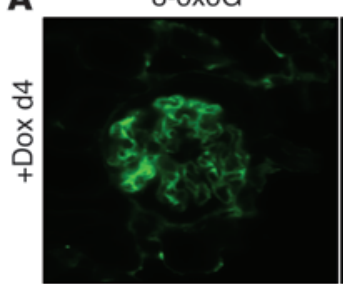

B

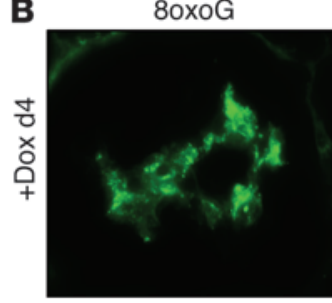

C
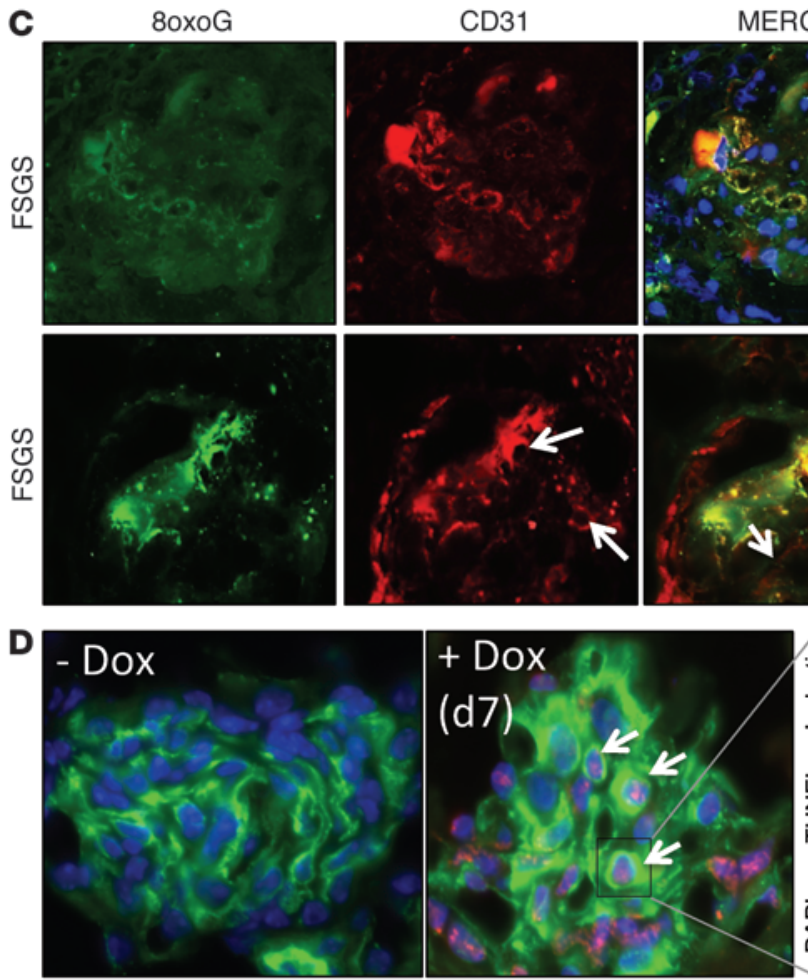

*

E

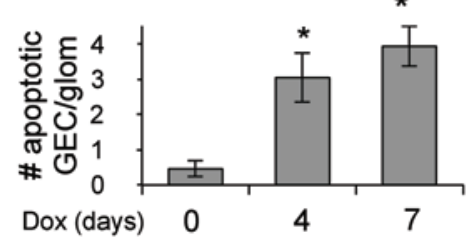

Thus, TGF $\beta$ R1 activation in podocytes rapidly upregulates preproendothelin-1 synthesis and EDN1 release in vivo and in vitro through SMAD-dependent signaling. Podocyte EDN1 release is associated with increased endothelial EDNRA expression and mitochondrial damage, consistent with paracrine podocyte-to-endothelial crosstalk mediated by EDN1/EDNRA. Indeed, EDN1/ EDNRA signaling in endothelial cells has been previously shown to cause oxidative injury $(38,39)$.

\section{Figure 4}

TGF- $\beta$ signaling in podocytes induces oxidative stress specifically in endothelial cells. (A) Representative doubleimmunofluorescence images of 8-oxoG (green), endothelial cell marker CD31 (red), and merge showing colocalization. (B) Representative double-immunofluorescence images of 8-oxoG (green), podocyte marker synaptopodin (red), and merge in glomeruli of a day 4 Dox-treated PodTgfbr1 mouse showing no colocalization in podocytes. (C) 8-oxoG (green) and endothelial cell marker CD31 (red) in glomeruli of kidney biopsies from subjects diagnosed with FSGS. Highermagnification images showing capillary loops are shown on the bottom row (arrows) (original magnification, $\times 63$ ). (D) Immunofluorescence staining showing TUNEL and isolectin localization with DAPI in untreated mice and mice treated with Dox for 7 days. Arrows depict nuclear TUNEL with DAPIand isolectin-positive endothelial cells. (E) Quantification of TUNEL- and isolectin-positive cells per glomeruli in untreated PodTgfbr1 mice and in PodTgfbr1 mice treated with Dox for 4 or 7 days ( ${ }^{*} P<0.05$; mean $\pm \mathrm{SD}$ ). Original magnification, $\times 63$ (A, B, C [bottom row], and D); ×40 (C [top row]).

EDN1/EDNRA-specific peptide antagonist BQ-123 or mitochondria-targeted antioxidant mitoTEMPO eliminates mitochondrial oxidative stress and dysfunction of endothelial cells and prevents podocyte defects and glomerular lesions in murine glomerular injury models. To test for functional consequences of EDN1/EDNRA podocyte-endothelial crosstalk, Dox-treated PodTgfbr 1 mice or AD-induced BALB/c mice received systemic administration of the EDN1/EDNRA antagonist BQ-123 (40), the mitochondrial-targeted potent antioxidant mitoTEMPO (41), or control vehicle. Compared with administration of vehicle, BQ-123 or mitoTEMPO administration eliminated the early endothelial 8-oxoG accumulation typical of day 4 of Dox treatment (Figure 6A). Inhibition of early 8-oxoG accumulation was associated with prevention of subsequent lesions of progressive glomerulosclerosis common after day 14 of Dox treatment in PodTgfbr 1 mice (Figure 6, A and B). Similar results were obtained in AD-induced BALB/c mice (Figure 6, E and F). BQ-123- or mitoTEMPO-mediated prevention of early endothelial mtDNA damage and subsequent glomerulosclerosis lesions was further associated with prevention of podocyte depletion (Figure 6C) and prevention of the rise of serum creatinine (Figure 6D) in Dox-treated PodTgfbr1 and AD-treated BALB/c mice (Figure 6, G and H). BQ-123 and mitoTEMPO treatment also ameliorated Dox- or AD-associated increase of urinary ACR and urinary 8-oxoG levels in both models (PodTgfbr1, Supplemental Figure 6, A and B, and AD-BALB/c, Figure 6, $\mathrm{C}$ and $\mathrm{D}$, respectively). Importantly, nuclear SMAD2/3 localization was not altered by the presence of BQ-123 or mitoTEMPO in Dox-fed PodTgfor 1 mice compared with that in controls (Supplemental Figure 6, E and F), suggesting persistent transgenic TGF $\beta$ R1 signaling in podocytes. Together, these results demonstrate that EDN1/EDNRA-mediated mitochondrial oxidative stress and dysfunction in endothelial cells are required for high-grade albuminuria, podocyte depletion, and glomerulosclerosis in both podocyte injury models.

EDN1-dependent podocyte-endothelial crosstalk induces endothelial mitochondrial oxidative stress and endothelial cell dysfunction in podocyteendothelial cell culture system. Conditionally immortalized POD cell 


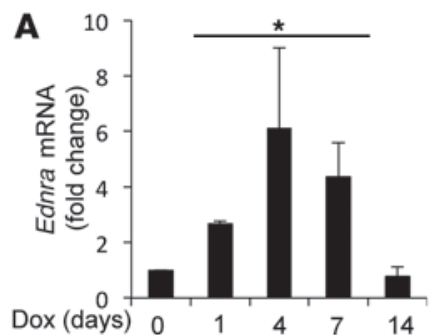

D
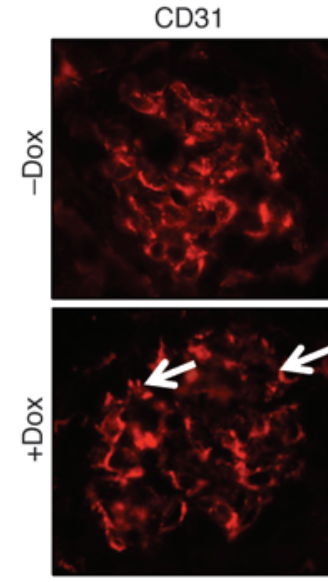

E

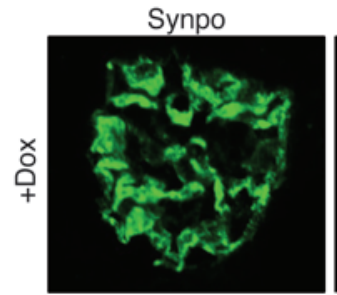

$\mathbf{F}$

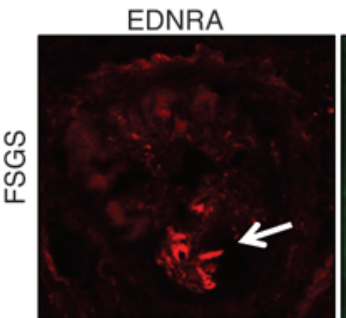

G

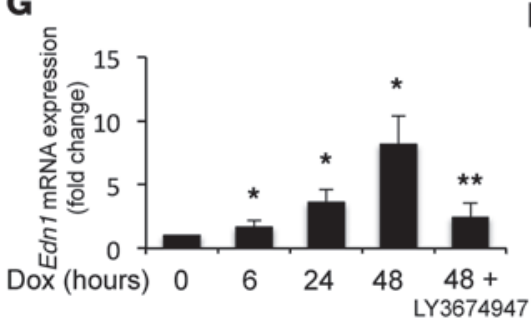

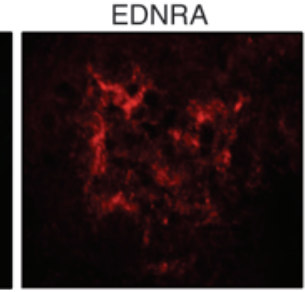

B -Dox

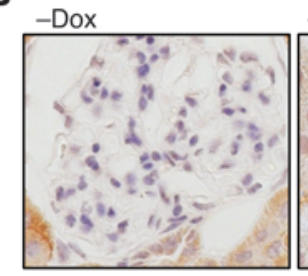

EDNRA
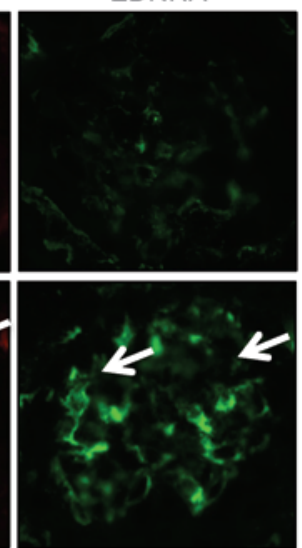

EDNRA

8-oxoG

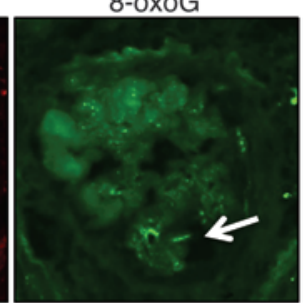

H $\widehat{\sum}_{0=0}$
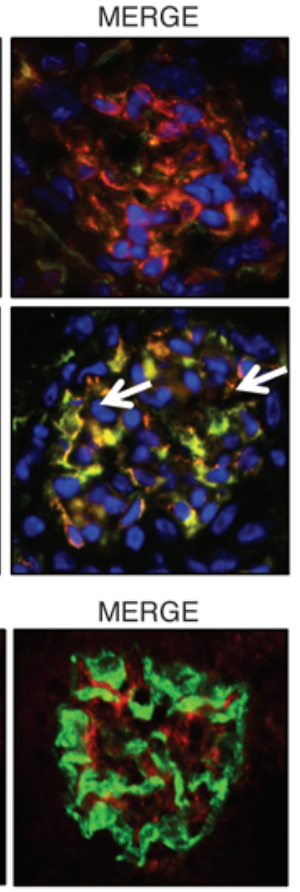

MERGE

MERGE

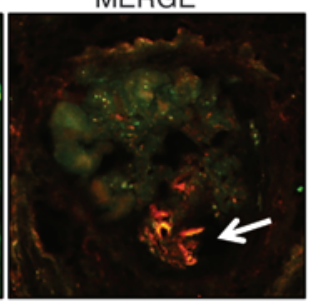

+ Dox (d1)

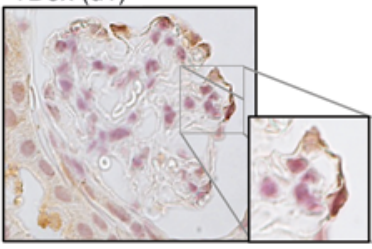

C

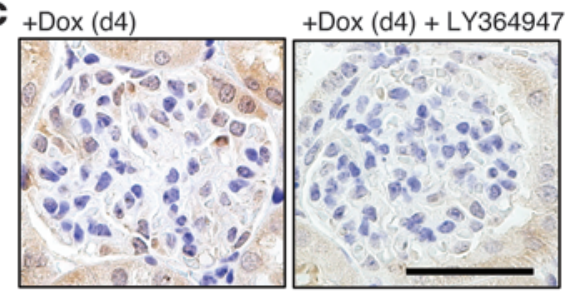

\section{Figure 5}

TGF- $\beta$ signaling in podocytes induces EDNRA specifically in endothelial cells. (A) Quantification by RT-PCR of Ednra mRNA in isolated glomeruli of untreated control PodTgfbr1 mice and PodTgfbr1 mice at day 1 to day 14 of Dox diet ( $n=3$ mice per group; mean \pm SEM; ${ }^{*} P<0.05$ versus controls). (B) Immunoperoxidase detection of preproendothelin-1 in control untreated PodTgfbr1 mice or mice with Dox treatment for 1 day (original magnification, $\times 100$ [inset]) or (C) 4 days with or without LY364947 (1 mg/kg). Scale bar: $50 \mu \mathrm{m}$. (D) Representative double-immunofluorescence detection of endothelial cell marker CD31 (red) and EDNRA (green) in glomeruli of untreated or day 7 Dox-treated PodTgfbr1 mice. (E) Synaptopodin (green) and EDNRA (red) in glomeruli of a day 7 Dox-treated PodTgfbr 1 mouse showing no colocalization in podocytes. (F) EDNRA (red) and 8-oxoG (green) in kidney biopsy from a subject diagnosed with FSGS. (G) mRNA expression of Edn1 and Ednra by POD cell line treated with Dox $(1 \mu \mathrm{g} / \mathrm{ml})$ without or with LY364947 $(3 \mu \mathrm{M})$ for $0,6,24$, and 48 hours. (H) Quantification by ELISA of EDN1 release in SN by POD cells treated with DOX for 6 to 48 hours without or with LY364947. Colocalization is indicated by arrows in $\mathbf{D}$ and $\mathbf{F}$. Mean \pm SEM of 3 independent experiments $(\mathbf{A}, \mathbf{G}$, and $\mathbf{H})$. ${ }^{*} P<0.05$, ${ }^{* *} P<0.01$ versus untreated controls. Original magnification, $\times 63(\mathbf{D}$ and $\mathbf{E}) ; \times 40(\mathbf{F})$. lines derived from PodTgfbr 1 mice were introduced above (see Figure 5, $\mathrm{G}$ and $\mathrm{H}$ ). POD cells (Dox-inducible Tgfbr $1^{A A D}$ podocytes) were either left untreated (controls) or treated with Dox or TGF- $\beta 1$ for 24 hours prior to collection of conditioned supernatants (SNs), which were identified as Control-PSNs, Dox-PSNs, and TGF- $\beta 1$ PSNs, respectively. When applied to murine glomerular endothelial cells (mGECs) in culture, Dox-PSN and TGF- $\beta 1$-PSN significantly upregulated Ednra mRNA, but not Edn1 mRNA, when compared with Control-PSN (Supplemental Figure 7A). Furthermore, when compared with Control-PSN, Dox-PSN markedly increased the number of mGECs expressing EDNRA cell surface protein (Supplemental Figure 7B). Compared with Control-PSN, Dox-PSN treatment significantly increased mitochondrial superoxide production (MitoSOX) (Figure 7A), oxidative mtDNA damage (8-oxoG) (Figure 7, B and C), and mtDNA lesions (Supplemental Figure 7C) in mGECs. Coincubation with BQ-123 or mitoTEMPO significantly 
A

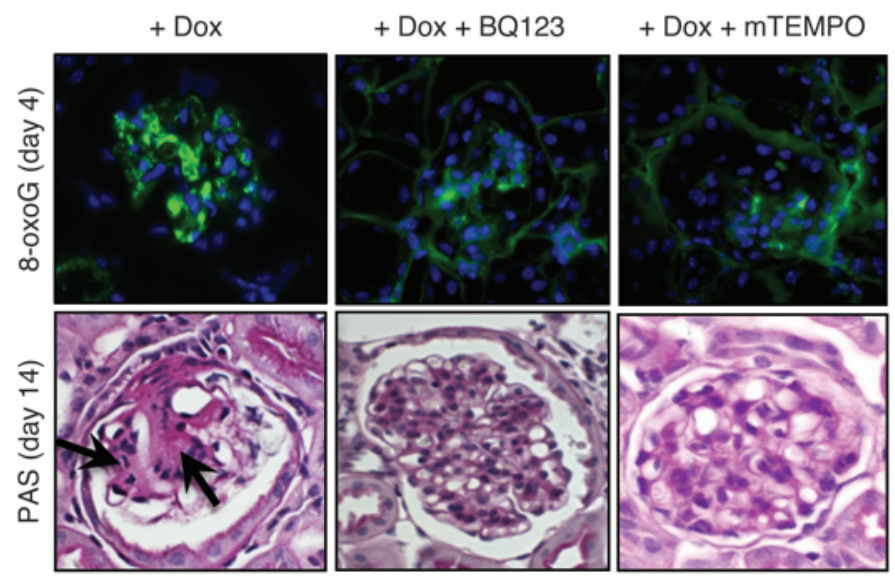

B

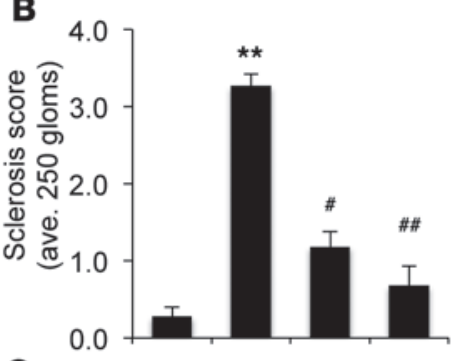

c
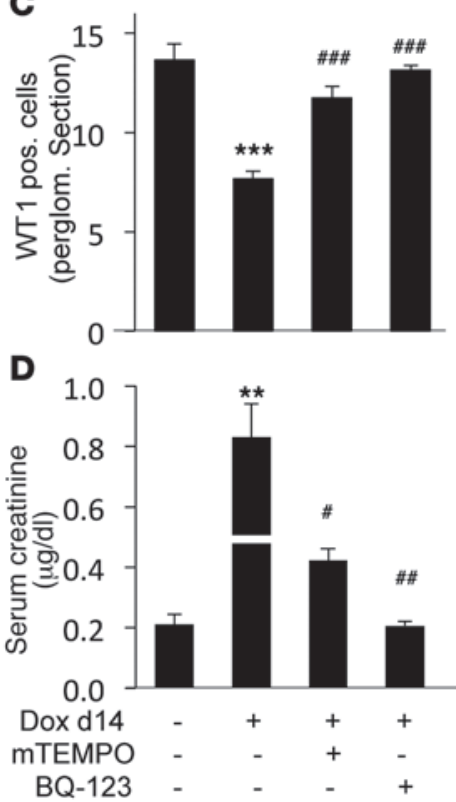

E

BALC/c + Adriamycin

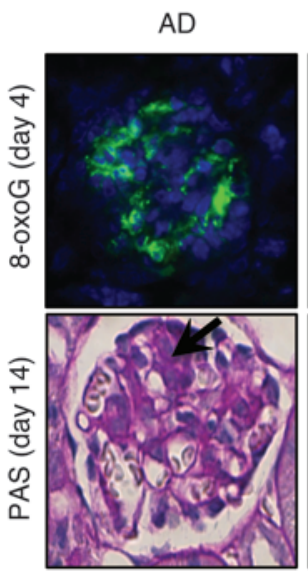

$\mathrm{AD}+\mathrm{BQ} 123$

AD+mTEMPO
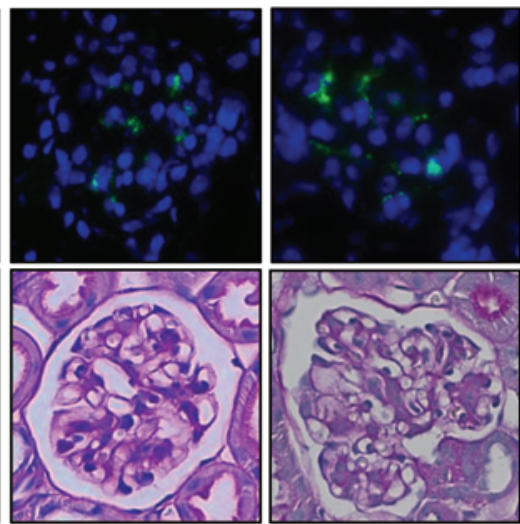

$\mathbf{F}$

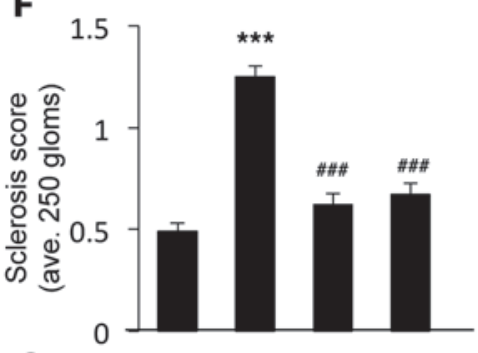

G
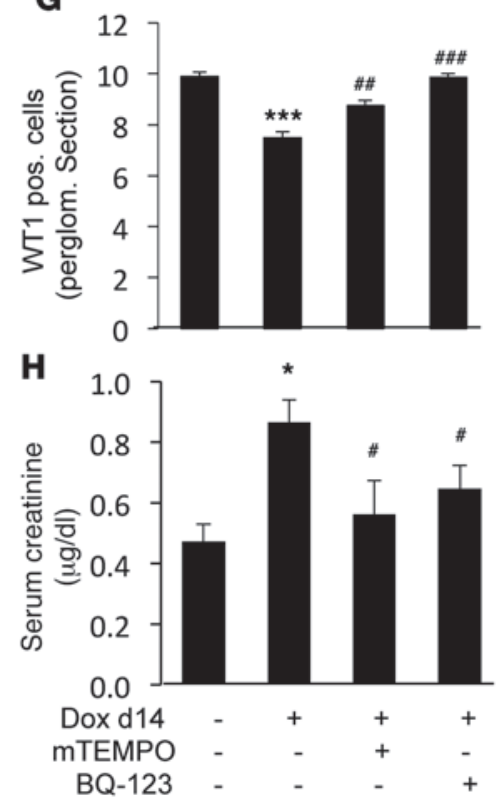

Figure 6

TGF- $\beta$ signaling in podocytes induces specific EDN1-mediated mitochondrial oxidative stress specifically in endothelial cells. (A) Immunofluorescence detection of mtDNA 8-oxoG in kidneys of day 4 Dox PodTgfbr1 mice, showing prominent glomerular staining; day 4 Dox PodTgfbr1 mice cotreated with BQ-123 (0.1 nM/kg/d); or day 4 Dox PodTgfbr1 mice cotreated with mitoTEMPO (1 mg/kg/d). PAS staining of day 14 Dox PodTgfbr1 kidneys, showing severe sclerosis (arrows), and day 14 Dox PodTgfbr1 kidneys cotreated with BQ-123 (0.1 nM/kg/d) or with mitoTEMPO (1 mg/kg/d). (B) Sclerosis index score (see Supplemental Methods). (C) Podocyte number and (D) serum creatinine levels of untreated PodTgfbr1 mice, Dox-treated PodTgfbr1 mice (day 14), or Dox-treated PodTgfbr1 mice cotreated with BQ-123 or mitoTEMPO $(n=5$; mean \pm SEM). (E) Immunofluorescence for 8-oxoG in kidneys of BALB/c mice after AD treatment, treatment with AD and BQ-123 (0.1 nM/kg/d), or treatment with $A D$ and mitoTEMPO $(1 \mathrm{mg} / \mathrm{kg} / \mathrm{d})$. PAS staining of glomeruli of BALB/c mice after AD treatment, showing severe sclerosis; after cotreatment with AD and BQ-123; or after cotreatment with AD and mitoTEMPO. (F) Sclerosis index score. (G) Podocyte number and (H) serum creatinine levels in BALB/c mice with or without AD treatment or with cotreatment with AD and BQ-123 or AD and mitoTEMPO $(n=6$; mean \pm SEM). (B, C, F, and G) Mean \pm SEM of at least 250 glomeruli from 5 to 8 mice per group. ${ }^{\star} P<0.05,{ }^{* \star} P<0.01,{ }^{* \star *} P<0.001$ versus control

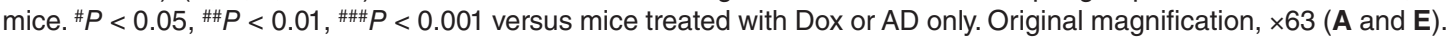


inhibited the induction of MitoSOX (Figure 7A) and 8-oxoG (Figure 7B) by Dox-PSN. Dox-PSN or TGF- $\beta$-PSN suppressed mRNA levels for selected mitochondrial respiratory complex genes in mGECs in vitro (Supplemental Figure day 7), consistent with their reduction, as observed in glomeruli from Dox-treated PodTgfbr1 mice in vivo (see Figure 3). Finally, the significant Dox-PSNinduced suppression of mitochondrial OCR in mGECs was prevented by coincubation with BQ-123 or mitoTEMPO (Figure 7D).

To inactivate $E d n 1$ gene product in POD cells and Ednra gene product in mGECs at a molecular level, we transfected POD cells with EDN1 siRNA or scrambled control siRNA (SCR siRNA). EDN1 siRNA and EDNRA siRNA efficiently (>85\%) knocked down Edn1 and Ednra mRNA (Supplemental Figure 8, A and B) and protein (Supplemental Figure 8, C and D), respectively. Induction of MitoSOX (Figure 7E) and mtDNA 8-oxoG staining (Figure 7F) by EDN1 or by Dox-PSN in mGECs was significantly reduced in EDNRA siRNA-transfected mGECs or in mGECs incubated with Dox-PSN from EDN1 siRNA-transfected POD cells (Figure 7, $\mathrm{E}$ and $\mathrm{F}$ ). Mitochondrial dysfunction in endothelial cells has previously been associated with endothelial dysfunction characterized by decreased bioavailability of nitric oxide and inhibition of NOS (42). Indeed, incubation of mGECs with Dox-PSN inhibited NOS to a comparable degree as the NOS inhibitor L-NAME, which was used as positive control (Figure 7G). Dox-PSN suppressed NOS in a dose-dependent manner, and this suppression was prevented by coincubation with BQ-123 or mitoTEMPO (Figure 7G). Together, these results demonstrate that EDN1 released by podocytes after TGF- $\beta 1$ or Dox stimulation signals through EDNRA on mGECs to induce mitochondrial oxidative stress, oxidative mtDNA damage, and mitochondrial dysfunction, resulting ultimately in hallmark endothelial dysfunction characterized by NOS inhibition and decreased NO bioavailability.

EDN1/EDNRA-induced endothelial cell dysfunction is necessary for a paracrine endothelial-podocyte signaling activity required for TGF $\beta R$ 1-associated podocyte apoptosis. Our in vivo results suggested that endothelial mitochondrial oxidative stress and endothelial dysfunction, likely induced by podocyte-derived EDN1 and endothelial cell EDNRA upregulation and activation, preceded and were required for manifestations of podocyte injury, including foot process effacement, podocyte apoptosis, and depletion. Based on these observations, we reasoned that SNs from EDN1- or Dox -PSN-stimulated mGECs may be required for POD apoptosis in our defined culture system. First, we confirmed that EDN1 treatment increased MitoSOX in mGECs (Supplemental Figure 9A). Incubation of POD cells with endothelial cell SN (ESN) from untreated control mGECs had no effect on POD apoptosis, irrespective of absence or presence of Dox (Supplemental Figure 9B). In contrast, ESN from EDN1-treated mGECs was required for increased Dox-induced POD apoptosis (Supplemental Figure 9B). Next, we used a Transwell coculture system for POD cell and mGEC coculture. Dox-induced TGF $\beta$ R 1 activation in POD cells increased MitoSOX in adjacent mGECs after 48 hours, and this paracrine effect was prevented by coincubation with BQ-123 or mitoTEMPO (Figure 8A). Increased MitoSOX in mGECs was essential for increased POD apoptosis after Dox stimulation, as demonstrated by prevention of POD apoptosis in the presence of BQ-123 or mitoTEMPO (Figure 8B). Direct EDN1 treatment of POD cells or incubation with control $\mathrm{mGEC} S \mathrm{SN}$ in the presence of Dox did not significantly change POD cell apoptosis (Figure 8C). POD cell apoptosis was significantly increased by incubation of Dox-treated POD cells with SN from EDN1-treated control siRNA-transfected mGECs (Figure 8C), while SN from EDN1treated EDNRA siRNA-transfected mGECs had no effect on POD cell apoptosis. Together, these in vitro results, from both direct coculture and media transfer studies, demonstrate consistently and at a molecular level that EDN1/EDNRA-mediated podocyteendothelial crosstalk causes endothelial dysfunction of mGECs, which was necessary and sufficient to increase podocyte apoptosis in TGF $\beta$ R1-activated (Dox-stimulated) POD cells.

\section{Discussion}

We report a novel and essential signaling crosstalk between glomerular podocytes and endothelial cells in progressive glomerulosclerosis (Figure 9). Various mechanisms of podocyte injury activate SMAD proteins associated with EDN1 precursor synthesis and EDN1 release. Paracrine EDN1/EDNRA signaling causes mitochondrial oxidative damage and dysfunction in glomerular endothelial cells. Endothelial mtDNA damage and dysfunctiondependent paracrine activities are required for TGF $\beta$ R1/SMAD-induced podocyte depletion and segmental glomerulosclerosis with nephrotic syndrome, elevated serum creatinine, and eventually decreased survival. The mechanism underlying podocyte apoptosis after endothelial dysfunction remains to be defined, although decreased NO, as shown in our in vitro studies, may be a culprit, given that loss of eNOS from glomerular endothelial cells has been recently demonstrated to affect podocyte function via activation of RhoA (43). More recently, in an eNOSdeficient model, glomerular endothelial cell injury was also shown to precede podocytes apoptosis after $\mathrm{AD}$ treatment, although a mechanism was not investigated (44). Interestingly, activation of podocytes also induced transient mesangial proliferation through mechanisms yet to be elucidated. This may be due to potential mitogenic effects of EDN1, effects on mesangial cells (45), or other secreted factors, although in our model, as well as AD- and Dicer-induced FSGS, most of the EDNRA receptors we found were in glomerular endothelial cells. Of note, mesangial proliferation rates had returned to baseline prior to onset of substantial podocyte loss in our model.

Podocytes control endothelial cell survival and glomerular homeostasis through paracrine VEGFA signaling (16). Glomerular VEGFA and VEGF receptor expression remained unchanged in PodTgfbr 1 mice and decreased only after severe podocyte depletion, albuminuria, and glomerulosclerosis were established, suggesting that in our model VEGF signaling is not involved in podocyteendothelial crosstalk until advanced podocyte depletion is established. Similarly to VEGF, EDN1 generated by activated podocytes is expected to diffuse across the basement membrane against the filtration flow to reach adjacent endothelial cells in capillary segments with stressed/injured podocytes.

Numerous reports implicate EDN1 in kidney injury and disease, including infusion in healthy humans (46) and rats (47), diabetes (48), or murine models with FSGS (49). EDN1 may affect podocytes directly, as shown by in vitro studies using serum samples from patients with preeclampsia, which stimulated EDN1 production by endothelial cells and resulted in nephrin shredding from podocytes via EDNRA (50). More recently, trials using EDN1 antagonists have shown reduction of proteinuria as well as improvements in peripheral endothelial function in patients with type 2 diabetes (51). Moreover, EDN1 has been shown to be produced by podocytes in vitro (52), consistent with our findings. EDN1 promotes mitochondrial 

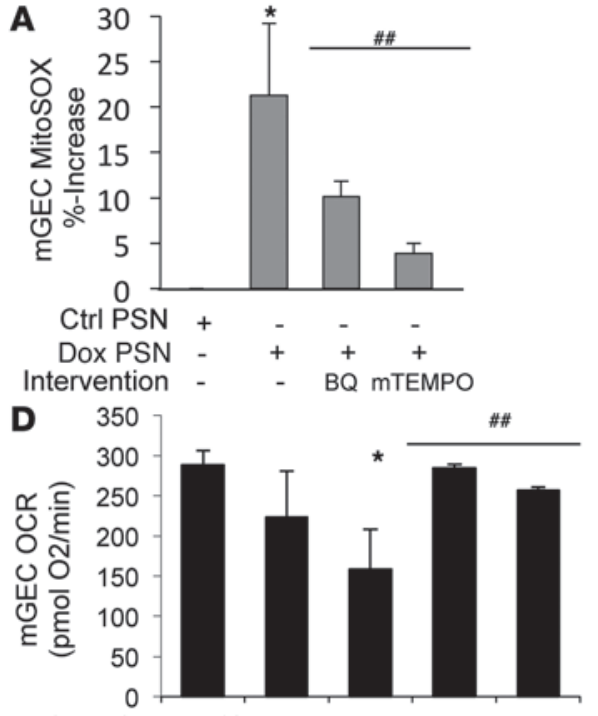

Ctrl PSN $100 \%$

Dox PSN - $50 \% \quad 100 \% \quad 100 \% 100 \%$

Intervention - $\quad-\quad-\quad B Q$ mTEMPO

\section{E}
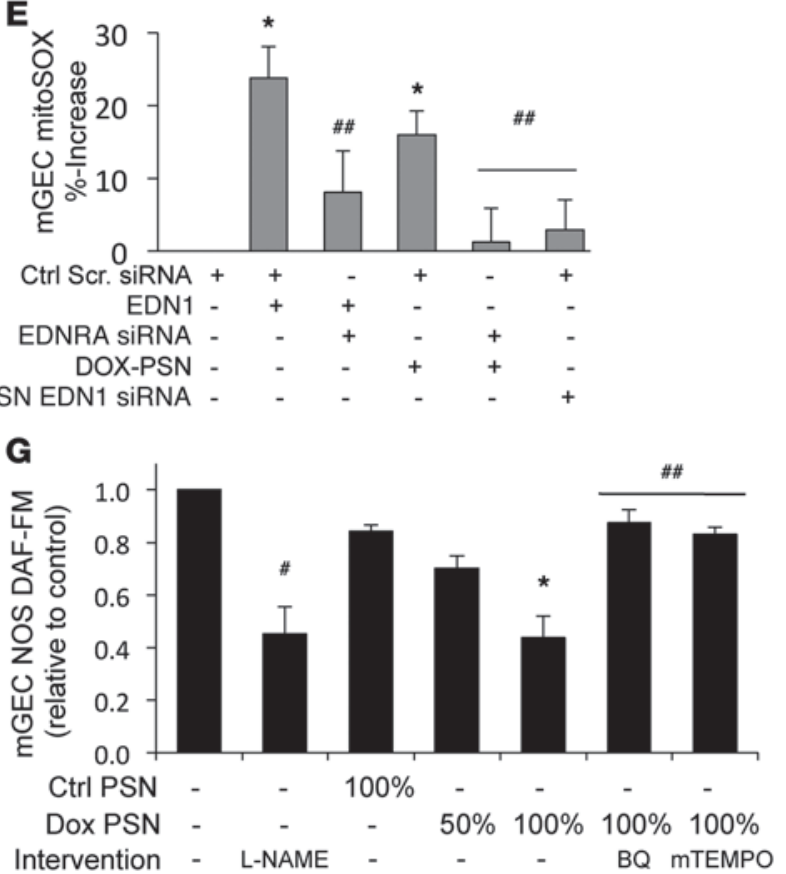
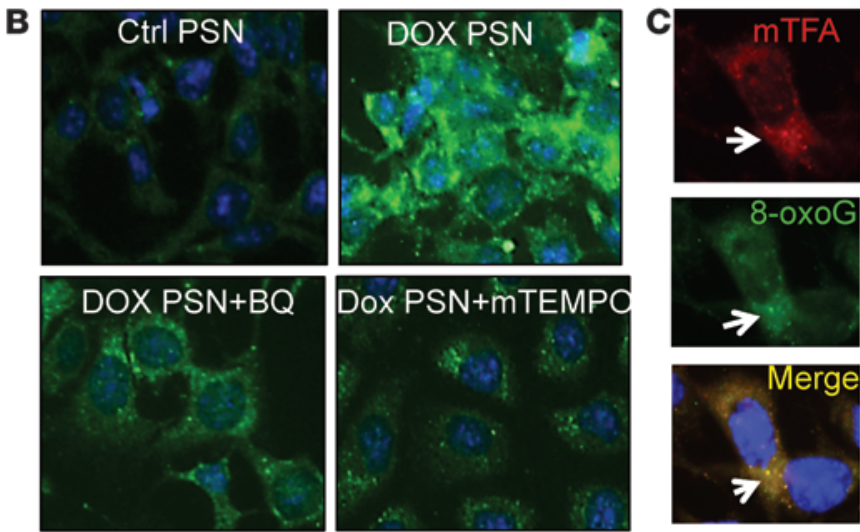
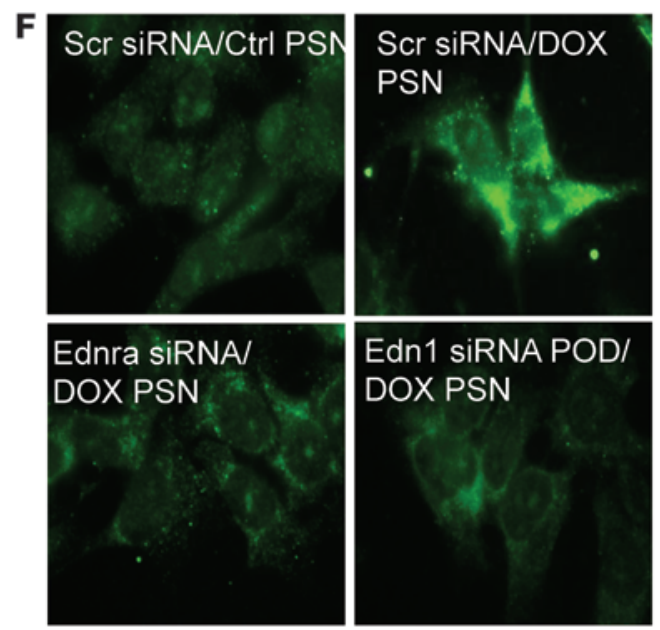

\section{Figure 7}

Validation of in vivo results using a defined PodTgfbr1-derived podocyte and in mGEC coculture system. (A) Percentage increase of MitoSOX bright fluorescent mGECs after culture with Control-PSN or Dox-PSN $(1 \mu \mathrm{g} / \mathrm{ml})$ for 24 hours in the absence or presence of BQ-123 (1 ng/ml) or mitoTEMPO $(5 \mu \mathrm{g} / \mathrm{ml})$. (B) Immunofluorescence detection of 8-oxoG in mGECs cultured as described in A. (C) Double-immunofluorescence detection of 8-oxoG (green) and mitochondrial transcription factor A (red) in mGECs cocultured in Dox-PSN (arrows indicates colocalization).

(D) OCR in mGECs cultured 24 hours with $100 \%$ Control-PSN or $50 \%$ or $100 \%$ Dox-PSN in the absence or presence of BQ-123 or mitoTEMPO. (E) Percentage increase of MitoSOX fluorescent mGECs transfected with control SCR siRNA or EDNRA siRNA and treated with EDN1 (200 nM), Dox-PSN, or Dox-PSN from EDN1 siRNA-transfected podocytes. (F) 8-oxoG immunofluorescence in mGECs transfected with control SCR siRNA or EDNRA siRNA and cultured 24 hours with either Control-PSN or Dox-PSN, as indicated. (G) NOS activity detected by DAF-FM fluorescence in mGECs in RPMI control media without or with L-NAME $(100 \mu \mathrm{M})$ or in mGECs cultured for 24 hours with $100 \%$ Control-PSN or $50 \%$ to $100 \%$ Dox-PSN in absence or presence of BQ-123 or mitoTEMPO. (A, D, E, and G) Each bar represents $n=3$; mean \pm SEM. ${ }^{*} P<0.05$ versus SN controls, ${ }^{\#} P<0.05$ versus RPMI controls, $\# \# P<0.05$ versus Dox. Cocultures performed in $10 \%$ FCS. Original magnification, $\times 100(B, C$, and $\mathbf{F})$. 


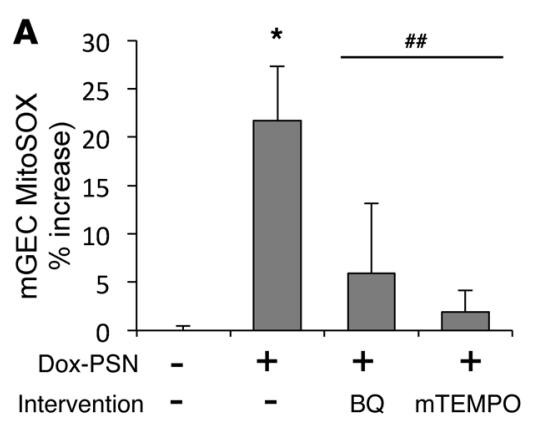

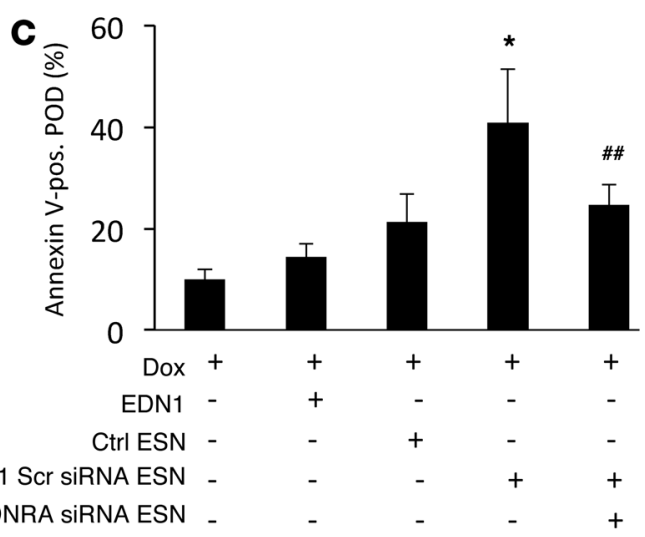

\section{Figure 8}

EDN1-mediated endothelial cell mitochondrial oxidative stress and dysfunction are required for podocyte apoptosis induced by TGF- $\beta / S M A D$ signaling in cell death. (A) Percentage of MitoSOX bright fluorescent mGECs after 48 hours transwell coculture with POD cells with or without Dox or Dox in the presence of BQ-123 $(1 \mathrm{ng} / \mathrm{ml})$ or mitoTEMPO $(5 \mu \mathrm{g} / \mathrm{ml})$. (B) Percentage apoptotic POD cells after coculture with mGECs in transwell inserts without or with Dox or Dox in the presence of BQ-123 $(1 \mathrm{ng} / \mathrm{ml})$ or mitoTEMPO $(5 \mu \mathrm{g} / \mathrm{ml})$ for 48 hours. (C) POD cells treated with Dox were treated directly with EDN1 or cultured with control mGEC SN (Ctl ESN), SN from EDN1-treated mGECs transfected with scrambled siRNA (EDN1 SCR siRNA ESN), or SN from EDN1-treated mGECs transfected with EDNRA siRNA (EDN1 EDNRA siRNA ESN), as indicated. Cocultures performed in $10 \%$ FCS. For all histograms, each bar represents $n=3$ independent experiments \pm SEM. ${ }^{*} P<0.05$ versus SN controls, $\# P<0.05$ versus RPMI controls, $\# P<0.05$ versus Dox.

impairment and cardiovascular dysfunction in hypertensive disease (53), and vascular oxidative stress in deoxycorticosterone acetate-salt (DOCA-salt) rats is associated with activation of the EDN1/EDNRA system (54). Also mitoTEMPO, a novel mitochondrial-targeted antioxidant (41), but not nontargeted TEMPOL, prevented mitochondrial superoxide in endothelial cells and lowered BP in mice made hypertensive with angiotensin II or DOCA-salt (55). Our results indicate that mechanisms of renal injury initiated by podocyte-derived EDN1 appear to be independent of systemic BP elevation

Currently, renin angiotensin system (RAS) antagonists provide the only class of compounds available to reduce proteinuria and slow loss of renal function (56). However, despite the widespread clinical use of RAS blockade, the prevalence of advanced stages of CKD continues to increase (1); hence, new therapies averting progression of CKD constitute an extraordinary unmet need in clinical practice. Trials of EDN1 receptor blockade have demonstrated efficacy in proteinuric glomerular diseases when added to standard RAS blockade, yet clinical development ceased, because these agents caused fluid retention $(57,58)$. However, specific EDN1 antagonists are currently in trials in patients with kidney disease. In light of our findings, selective targeting of the mitochondrial dysfunctioninducing activity of EDN1/EDNRA in glomerular endothelial cells may provide a promising therapeutic approach for podocyteinitiated glomerular diseases. Traditional antioxidants, including TEMPOL, have limited mitochondrial accumulation (59). In contrast, agents, such as mitoTEMPO, are concentrated in the mitochondrial matrix by 1,000-fold because of their positive charge (41). We propose that new ultrapotent mitochondrial-targeted antioxidants, such as mitoTEMPO and others, warrant consideration as a novel approach to prevent progression of glomerulosclerosis.

In summary, our findings have profound implications for the current podocyte depletion paradigm and for novel treatment opportunities in common glomerular diseases manifesting with segmental glomerulosclerosis. To our knowledge, this is the first report demonstrating that progressive segmental glomeruloscle- rosis with characteristic loss of podocytes and massive proteinuria can be mitigated by prevention of mitochondrial damage specifically in glomerular endothelial cells by inhibition of EDN1 signaling via EDNRA and/or by mitochondrial-targeted ROS scavenging. The novel podocyte-endothelium-podocyte crosstalk occurring in the early/reversible stage may underlie certain forms of kidney disease and open new therapeutic avenues to protect these patients from further renal damage.

\section{Methods}

\section{Mice and cell lines}

PodTgfor 1 mice were generated as described in the Supplemental Methods (see also Supplemental Figure 1). Dox chow contained 2,000 ppm Dox in regular AIN-76A food pellets (Research Diets Inc.).

Male BALB/c mice were injected with AD (10 mg/kg; Sigma-Aldrich), as previously reported (37), or saline (controls) in the tail vein at day 0 . Mice were given 2 daily $2 \mathrm{ml}$ intraperitoneal injections of an isotonic solution $(69.4 \mathrm{mM}$ glucose, $77 \mathrm{mM} \mathrm{NaCl}$ ) to prevent weight loss. Weight was monitored daily.

MitoTEMPO (Enzo Life Sciences International), BQ-123 (SigmaAldrich), or saline were delivered by subcutaneous Alzet miniosmotic pumps (model 2002). LY364947 (1 mg/kg) was administered daily by intraperitoneal injection.

The generation of conditionally immortalized Nphs2-rtTA.Tgfbr $1^{\text {AAD }}$ podocytes is described in the Supplemental Methods. WT, Smad2 KO, Smad2/3 DKO podocytes and POD cells and conditionally immortalized mGECs were maintained in RPMI- 1640 containing 10\% FCS under either permissive conditions $\left(33^{\circ} \mathrm{C}\right.$ with IFN- $\gamma, 10$ units per $\left.\mathrm{ml}\right)$ or nonpermissive conditions $\left(37^{\circ} \mathrm{C}\right.$ without IFN- $\left.\gamma\right)$.

Transwell cocultures were performed in collagen-I-coated 6-well plates and $0.4 \mu \mathrm{m} \times 24 \mathrm{~mm}$ inserts (Costar).

\section{Isolation of glomeruli}

The procedure for isolation of glomeruli was based on a previously published method (36). 


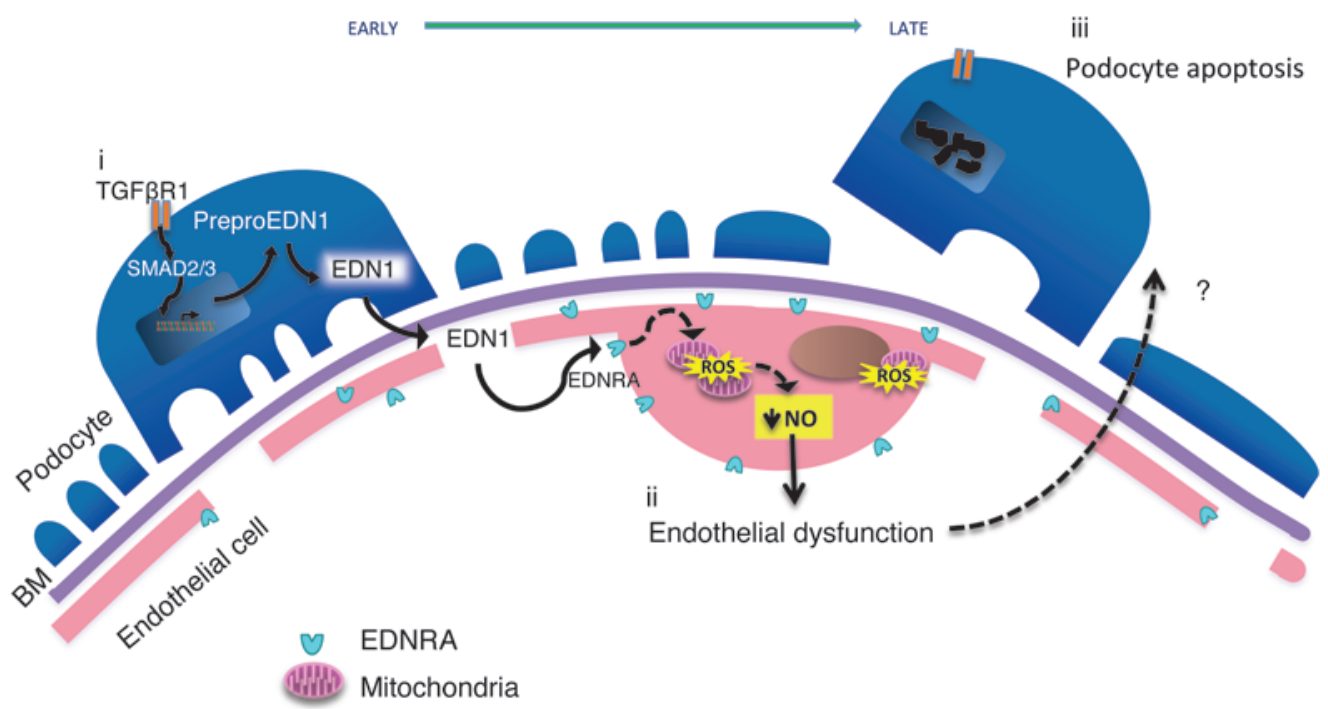

\section{Figure 9}

Working model of podocyte-glomerular endothelial cell crosstalk. (i) Podocyte selective injury by TGF $\beta$ R1 activation (or loss of miRNA [Dicer KO] or treatment with $A D$ ) results in SMAD2/3-mediated preproendothelin-1 synthesis and release of EDN1 as well as increased EDNRA on cell surface of glomerular endothelial cells. (ii) Podocyte-derived EDN1 acts on endothelial cells with increased EDNRA expression to induce mitochondrial ROS, mtDNA damage, mitochondrial dysfunction, and endothelial dysfunction with decreased NO synthesis. (iii) Factors that are either applied or deficient in dysfunctional endothelial cells are required for foot process effacement, apoptosis, and/or detachment of podocytes.

\section{Serum creatinine}

Serum creatinine was measured by acetonitrile deproteinization, followed by isocratic cation exchange HPLC, as previously described (60).

\section{Albuminuria \\ Urinary albumin and creatinine were measured using mouse albumin- specific ELISA and Creatinine Companion Kits (Exocell Laboratories).}

\section{Sclerosis index}

The sclerosis index scoring to evaluate glomerulosclerosis was performed as previously described (61).

\section{$O C R$ and extracellular acidification rate measurement}

Glomerular and tubular fractions from PodTgfbr1 mice were plated in collagen-I-coated XF24-well microplates (Seahorse Bioscience). OCR and extracellular acidification rate were analyzed with the Seahorse Bioscience XF24 Extracellular Flux Analyzer in basal conditions and after injections of $1 \mu \mathrm{M}$ FCCP [carbonyl cyanide 4-(trifluoromethoxy) phenylhydrazone].

\section{Flow cytometry}

Fluorescence assay for mitochondrial ROS. Fluorescence assay for mitochondrial ROS was measured in cells by MitoSOX (Invitrogen), per the manufacture's instructions.

Cell surface expression. Cell surface expression was determined, as previously described (62), with anti-EDNRA antibody (Santa Cruz) and with FITC-conjugated anti-rabbit antibody (Molecular Probes).

Viability and apoptosis. Viability and apoptosis 2-color flow cytometry was performed as described previously (62) using Annexin V-PE with 7AAD (BD Pharmingen). 10,000 cells were assayed by a BD FACSCanto (BD Biosciences).

\section{Quantitative PCR}

RT-PCR. Total RNA was prepared from glomeruli and cell lysates using Qiagen RNeasy Mini Columns (Qiagen) and then reverse transcribed with
SuperScript II Reverse Transcriptase (Invitrogen). cDNA amplification was performed in an ABI Prism 7900HYT Sequence Detection System and evaluated using S.D.S 2.0 software (Applied Biosystems). Normalization was performed using the murine $\beta$-actin gene. Primer sequences are listed in Supplemental Table 3.

Long PCR and lesion frequency. Total DNA was isolated using Qiagen DNeasy Mini Columns (Qiagen). DNA lesion frequencies were calculated as described previously (63).

\section{Microscopy}

PAS. PAS staining was performed on PFA-perfused, paraffin-embedded kidney sections using standard protocols.

Immunohistochemistry. Paraffin-embedded kidney sections were immunostained with anti-Nitrotyrosine, clone 2A8.2 (Millipore) or preproendothelin-1 (Abcam) and biotinylated anti-mouse immunoglobulins (Vectastain Kit; Vector Labs). 3,3'-Diaminobenzidine was used for visualization and hematoxylin was used as counterstain.

Immunofluorescence. 8-oxoG staining on PFA-perfused frozen sections or cells on coverslips was done as previously described (64) using anti-8-oxoG monoclonal antibody (N45.1; Japan Institute for the Control of Aging). Sections were also stained with goat anti-synaptopodin, rabbit anti-WT1, rabbit anti-ETA, and goat anti-mtTFA antibodies (Santa Cruz) or rat monoclonal anti-CD31 (MEC 7.46; Abcam).

Tunnel assay. Apoptotic nuclei in tissue sections were detected using DAPI $(1 \mu \mathrm{g} / \mathrm{ml})$ and WT1 or DAPI and isolectin with Apoptosis Detection Kit Apoptag Red (Millipore-Chemicon). Some sections were stained with Alexa Fluor 488 Isolectin GS-IB4 (Life Technologies).

Electron microscopy. The cortices from perfused kidneys were sliced into 1 - $\mathrm{mm}^{3}$ cubes and fixed in $2 \%$ glutaraldehyde solution, followed by standard protocols.

Quantitative digital image analysis. Images were taken as TIFF files with a Zeiss Axioplan2 equipped with a Q-imaging MP3.3 RTV color camera running QED capture software. Quantification was performed by MetaMorph 6.3r3 software (Molecular Devices). 
Superresolution structured illumination microscopy. Superresolution structured illumination microscopy was performed on an ELYRA PS.1 microscope from Zeiss.

\section{Human renal biopsies}

Paraffin-embedded human renal biopsy samples of 6 controls and 10 patients with focal segmental glomerulosclerosis were obtained from Vivette D'Agati. The study of human renal tissues was performed according to the Columbia University IRB protocol no. AAAE0986, entitled "Research with archival renal tissues. For patient information at the time of biopsy, see Supplemental Table 2.

\section{HPLC}

Urine 8-oxodG was measured with electrochemical detection $(65,66)$ using a Waters amperometric detector with a glassy carbon working electrode and $\mathrm{Ag} / \mathrm{AgCl}$ reference electrode. The oxidation potential was $0.4 \mathrm{~V}$. Levels of 8-oxodG were expressed relative to urine creatinine.

\section{EDN1 assay}

The EDN1 Enzyme Immunosorbent Assay Kit (Cayman Chemical) was used to detect EDN1 in cell culture-conditioned media and EDN1 was measured with a VICTOR3 V Multilabel Counter (450 nm; Perkin Elmer). Conditioned media samples were concentrated using SPE Bond Elute columns (Agilent).

\section{NOS}

NOS activity by live cells was determined by diaminofluorescein-2 diacetate using the NOS Detection Kit (Cell Technologies).

\section{Gene silencing}

RNA interference experiments in cells were performed after transfection of
EDNRA or EDN1 siRNA (10-20 nM; Silencer Select Invitrogen) or scrambled sequence siRNA, using Lipofectamine 2000 (Invitrogen).

\section{Statistics}

Results are expressed as mean \pm SD or mean \pm SEM, and some data were analyzed by 1-way ANOVA, followed by post-hoc $t$ test, using Dunnett's method (multiple comparisons versus control). Data were considered statistically significant when $P<0.05$.

\section{Study approval}

All animal protocols and procedures were approved by IACUC at the Mount Sinai School of Medicine. Human samples were collected under an IRB-approved protocol (no. AAAE0986) from archived kidney biopsies at Columbia Presbyterian Medical Center.

\section{Acknowledgments}

The authors thank Joan Massague for providing the Tgfbr $1^{A A D}$ mutant cDNA and Jeffry B. Kopp for providing the Nphs2-rtTA transgenic mice and valuable discussions. This work was supported by NIH grants 5U01DK060995, 5R01DK056077, 5R01DK060043, and 1R01DK097253-01A1 (to E.P. Bottinger).

Received for publication May 28, 2013, and accepted in revised form January 2, 2014.

Address correspondence to: Erwin Bottinger, The Charles Bronfman Institute for Personalized Medicine, Icahn School of Medicine at Mount Sinai, One Gustave Levy Place, Box 1003, New York, New York 10029, USA. Phone: 212.241.0800; Fax: 212.849.2643; E-mail: erwin.bottinger@mssm.edu.
1. United States Renal Data System. USRDS 2011 Annual Data Report. In: NIH, ed. Atlas of Chronic Kidney Disease and End-Stage Renal Disease in the United States. Bethesda, Maryland, USA: National Institute of Diabetes and Digestive and Kidney Diseases; 2011.

2. Shlipak MG, et al. Cardiovascular mortality risk in chronic kidney disease: comparison of traditional and novel risk factors. JAMA. 2005; 293(14):1737-1745.

3. Agarwal S, Shlipak MG, Kramer H, Jain A, Herrington DM. The association of chronic kidney disease and metabolic syndrome with incident cardiovascular events: multiethnic study of atherosclerosis. Cardiol Res Pract. 2012;2012:806102.

4. D’Agati VD, Kaskel FJ, Falk RJ. Focal segmental glomerulosclerosis. N Engl J Med. 2011; 365(25):2398-2411.

5. Fogo AB, Kon V. The glomerulus - a view from the inside - the endothelial cell. Int J Biochem Cell Biol. 2010;42(9):1388-1397.

6. Jarad G, Miner JH. Update on the glomerular filtration barrier. Curr Opin Nephrol Hypertens. 2009;18(3):226-232.

7. Wiggins RC. The spectrum of podocytopathies: a unifying view of glomerular diseases. Kidney Int. 2007;71(12):1205-1214.

8. Kriz W, Gretz N, Lemley KV. Progression of glomerular diseases: is the podocyte the culprit? Kidney Int. 1998;54(3):687-697.

9. Mundel P, Shankland SJ. Podocyte biology and response to injury. J Am Soc Nephrol. 2002; 13(12):3005-3015.

10. Mundel P, Reiser J. Proteinuria: an enzymatic disease of the podocyte? Kidney Int. 2010;77(7):571-580.

11. Patrakka J, Tryggvason K. New insights into the role of podocytes in proteinuria. Nat Rev Nephrol.
2009;5(8):463-468.

12. Bottinger EP. TGF- $\beta$ in renal injury and disease. Semin Nephrol. 2007;27(3):309-320.

13. Haraldsson B, Nystrom J. The glomerular endothelium: new insights on function and structure. Curr Opin Nephrol Hypertens. 2012;21(3):258-263.

14. Ballermann BJ. Contribution of the endothelium to the glomerular permselectivity barrier in health and disease. Nephron Physiol. 2007;106(2):19-25.

15. Schlondorff D, Banas B. The mesangial cell revisited: no cell is an island. J Am Soc Nephrol. 2009; 20(6):1179-1187.

16. Sison $\mathrm{K}$, et al. Glomerular structure and function require paracrine, not autocrine, VEGF-VEGFR-2 signaling. J Am Soc Nephrol. 2010;21(10):1691-1701.

17. Jeansson M, et al. Angiopoietin-1 is essential in mouse vasculature during development and in response to injury. $J$ Clin Invest. 2011; 121(6):2278-2289.

18. Haraldsson B, Jeansson M. Glomerular filtration barrier. Curr Opin Nephrol Hypertens. 2009;18(4):331-335.

19. Jeansson M, Bjorck K, Tenstad O, Haraldsson B. Adriamycin alters glomerular endothelium to induce proteinuria. J Am Soc Nephrol. 2009;20(1):114-122.

20. Schiffer M, et al. Apoptosis in podocytes induced by TGF- $\beta$ and Smad7. J Clin Invest. 2001;108(6):807-816.

21. Kim JH, Kim BK, Moon KC, Hong HK, Lee HS. Activation of the TGF- $\beta /$ Smad signaling pathway in focal segmental glomerulosclerosis. Kidney Int. 2003;64(5):1715-1721.

22 . Abbate $M$, et al. Transforming growth factor- $\beta 1$ is up-regulated by podocytes in response to excess intraglomerular passage of proteins: a central pathway in progressive glomerulosclerosis. Am J Pathol. 2002;161(6):2179-2193.

23. Shankland SJ, et al. Differential expression of transforming growth factor-b isoforms and receptors in experimental membranous nephropathy. Kidney Int. 1996;50(1):116-124.

24. Tojo A, Asaba K, Onozato ML. Suppressing renal NADPH oxidase to treat diabetic nephropathy. Expert Opin Ther Targets. 2007;11(8):1011-1018.

25. Wahab NA, et al. Glomerular expression of thrombospondin-1, transforming growth factor beta and connective tissue growth factor at different stages of diabetic nephropathy and their interdependent roles in mesangial response to diabetic stimuli. Diabetologia. 2005;48(12):2650-2660.

26. Patek CE, et al. Murine Denys-Drash syndrome: evidence of podocyte de-differentiation and systemic mediation of glomerulosclerosis. Hum Mol Genet. 2003;12(18):2379-2394.

27. Shigehara $T$, et al. Inducible podocyte-specific gene expression in transgenic mice. J Am Soc Nephrol. 2003;14(8):1998-2003.

28. Siegel PM, Shu W, Cardiff RD, Muller WJ, Massague $J$. Transforming growth factor $\beta$ signaling impairs Neu-induced mammary tumorigenesis while promoting pulmonary metastasis. Proc Natl Acad Sci U S A. 2003;100(14):8430-8435.

29. Shigehara T, et al. Inducible podocyte-specific gene expression in transgenic mice. J Am Soc Nephrol. 2003;14(8):1998-2003.

30. Derynck R, Zhang Y, Feng XH. Smads: transcriptional activators of TGF-beta responses. Cell. 1998; 95(6):737-740.

31. Wharram BL, et al. Podocyte depletion causes glomerulosclerosis: diphtheria toxin-induced podocyte depletion in rats expressing human diphtheria toxin receptor transgene. J Am Soc Nephrol. 2005;16(10):2941-2952.

32. Satoh $\mathrm{M}$, et al. NAD $(\mathrm{P}) \mathrm{H}$ oxidase and uncoupled nitric oxide synthase are major sources of glo- 
merular superoxide in rats with experimental diabetic nephropathy. Am J Physiol Renal Physiol. 2005;288(6):F1144-F1152.

33. Dijkstra G, et al. Expression of nitric oxide synthases and formation of nitrotyrosine and reactive oxygen species in inflammatory bowel disease. J Pathol. 1998;186(4):416-421.

34. Daehn I, Brem R, Barkauskaite E, Karran P. 6-Thioguanine damages mitochondrial DNA and causes mitochondrial dysfunction in human cells. FEBS Lett. 2011;585(24):3941-3946.

35. Kasai H, Nishimura S. Hydroxylation of deoxyguanosine at the C-8 position by ascorbic acid and other reducing agents. Nucleic Acids Res. 1984;12(4):2137-2145.

36. Shi S, et al. Podocyte-selective deletion of dicer induces proteinuria and glomerulosclerosis. $\mathrm{J} \mathrm{Am}$ Soc Nephrol. 2008;19(11):2159-2169.

37. Zheng Z, et al. A Mendelian locus on chromosome 16 determines susceptibility to doxorubicin nephropathy in the mouse. Proc Natl Acad Sci US A. 2005;102(7):2502-2507.

38. Callera GE, Tostes RC, Yogi A, Montezano AC, Touyz RM. Endothelin-1-induced oxidative stress in DOCA-salt hypertension involves NADPH-oxidase-independent mechanisms. Clin Sci (Lond). 2006;110(2):243-253.

39. Deng W, Baki L, Baumgarten CM. Endothelin signalling regulates volume-sensitive $\mathrm{Cl}$ - current via NADPH oxidase and mitochondrial reactive oxygen species. Cardiovasc Res. 2010;88(1):93-100.

40. Eguchi S, Hirata Y, Ihara M, Yano M, Marumo F. A novel ETA antagonist (BQ-123) inhibits endothelin-1-induced phosphoinositide breakdown and DNA synthesis in rat vascular smooth muscle cells. FEBS Lett. 1992;302(3):243-246.

41. Trnka J, Blaikie FH, Smith RA, Murphy MP. A mitochondria-targeted nitroxide is reduced to its hydroxylamine by ubiquinol in mitochondria. Free Radic Biol Med. 2008;44(7):1406-1419.

42. Montezano AC, Touyz RM. Reactive oxygen species and endothelial function--role of nitric oxide synthase uncoupling and Nox family nicotinamide adenine dinucleotide phosphate oxidases. Basic Clin Pharmacol Toxicol. 2012;110(1):87-94.

43. Yuen DA, et al. eNOS deficiency predisposes podocytes to injury in diabetes. J Am Soc Nephrol. 2012;23(11):1810-1823.

44. Sun YB, Qu X, Zhang X, Caruana G, Bertram JF, Li J. Glomerular endothelial cell injury and damage precedes that of podocytes in adriamycin-induced nephropathy. PLoS One. 2013;8(1):e55027.

45. Simonson MS, et al. Endothelin stimulates phospholipase $\mathrm{C}, \mathrm{Na}+/ \mathrm{H}+$ exchange, c-fos expression, and mitogenesis in rat mesangial cells. J Clin Invest. 1989;83(2):708-712.

46. Sorensen SS, Madsen JK, Pedersen EB. Systemic and renal effect of intravenous infusion of endothelin-1 in healthy human volunteers. Am J Physiol. 1994;266(3 pt 2):F411-F418.

47. Badr KF, Murray JJ, Breyer MD, Takahashi K, Inagami T, Harris RC. Mesangial cell, glomerular and renal vascular responses to endothelin in the rat kidney. Elucidation of signal transduction pathways. J Clin Invest. 1989;83(1):336-342.

48. Shin SJ, Lee YJ, Tsai JH. The correlation of plasma and urine endothelin-1 with the severity of nephropathy in Chinese patients with type 2 diabetes. Scand J Clin Lab Invest. 1996;56(6):571-576.

49. Chen HC, Guh JY, Chang JM, Tsai JC, Hwang SJ, Lai YH. Plasma and urinary endothelin-1 in focal segmental glomerulosclerosis. J Clin Lab Anal. 2001; 15(2):59-63.

50. Collino F, et al. Preeclamptic sera induce nephrin shedding from podocytes through endothelin-1 release by endothelial glomerular cells. Am J Physiol Renal Physiol. 2008;294(5):F1185-F1194.

51. Rafnsson A, Bohm F, Settergren M, Gonon A, Brismar K, Pernow J. The endothelin receptor antagonist bosentan improves peripheral endothelial function in patients with type 2 diabetes mellitus and microalbuminuria: a randomised trial. Diabetologia. 2012;55(3):600-607.

52 . Morigi $\mathrm{M}$, et al. In response to protein load podocytes reorganize cytoskeleton and modulate endothelin-1 gene: implication for permselective dysfunction of chronic nephropathies. Am J Pathol. 2005;166(5):1309-1320.

53. Yuki K, Suzuki T, Katoh S, Kakinuma Y, Miyauchi T, Mitsui Y. Endothelin-1 stimulates cardiomyocyte injury during mitochondrial dysfunction in culture. Eur J Pharmacol. 2001;431(2):163-170.
54. Callera GE, Bendhack LM. Mechanisms underlying the contractile response to endothelin- 1 in the rat renal artery. Pharmacology. 2003;68(3):131-139.

55. Dikalova AE, et al. Therapeutic targeting of mitochondrial superoxide in hypertension. Circ Res. 2010; 107(1):106-116.

56. Taal MW, Brenner BM. Renoprotective benefits of RAS inhibition: from ACEI to angiotensin II antagonists. Kidney Int. 2000;57(5):1803-1817.

57. Barton M. Endothelin antagonism and reversal of proteinuric renal disease in humans. Contrib Nephrol. 2011;172:210-222.

58. Kohan DE. Endothelin, hypertension and chronic kidney disease: new insights. Curr Opin Nephrol Hypertens. 2010;19(2):134-139.

59. Murphy MP, Smith RA. Drug delivery to mitochondria: the key to mitochondrial medicine. $A d v$ Drug Deliv Rev. 2000;41(2):235-250.

60. Yuen PS, Dunn SR, Miyaji T, Yasuda H, Sharma K, Star RA. A simplified method for HPLC determination of creatinine in mouse serum. Am J Physiol Renal Physiol. 2004;286(6):F1116-F1119.

61. Ma LJ, Fogo AB. Model of robust induction of glomerulosclerosis in mice: importance of genetic background. Kidney Int. 2003;64(1):350-355.

62. Daehn IS, Varelias A, Rayner TE. T-lymphocyte-induced, Fas-mediated apoptosis is associated with early keratinocyte differentiation. Exp Dermatol. 2010; 19(4):372-380.

63. Ayala-Torres S, Chen Y, Svoboda T, Rosenblatt J, Van Houten B. Analysis of gene-specific DNA damage and repair using quantitative polymerase chain reaction. Methods. 2000;22(2):135-147.

64. Ohno M, Oka S, Nakabeppu Y. Quantitative analysis of oxidized guanine, 8-oxoguanine, in mitochondrial DNA by immunofluorescence method. Methods Mol Biol. 2009;554:199-212.

65. Fraga CG, Shigenaga MK, Park JW, Degan P, Ames $\mathrm{BN}$. Oxidative damage to DNA during aging: 8-hydroxy-2'-deoxyguanosine in rat organ DNA and urine. Proc Natl Acad Sci U S A. 1990;87(12):4533-4537.

66. Germadnik D, Pilger A, Rudiger HW. Assay for the determination of urinary 8 -hydroxy-2'-deoxyguanosine by high-performance liquid chromatography with electrochemical detection. JChromatogr B Biomed Sci Appl. 1997;689(2):399-403. 